\title{
Soft Rough Approximation Operators and Related Results
}

\author{
Zhaowen Li, ${ }^{1}$ Bin Qin, ${ }^{2}$ and Zhangyong $\mathrm{Cai}^{3}$ \\ ${ }^{1}$ School of Science, Guangxi University for Nationalities, Nanning, Guangxi 530006, China \\ ${ }^{2}$ School of Information and Statistics, Guangxi College of Finance and Economics, Nanning, Guangxi 530003, China \\ ${ }^{3}$ Department of Mathematics, Guangxi Teachers College, Nanning, Guangxi 530023, China
}

Correspondence should be addressed to Zhaowen Li; lizhaowen8846@126.com

Received 2 August 2012; Revised 21 January 2013; Accepted 22 January 2013

Academic Editor: Hui-Shen Shen

Copyright (C) 2013 Zhaowen Li et al. This is an open access article distributed under the Creative Commons Attribution License, which permits unrestricted use, distribution, and reproduction in any medium, provided the original work is properly cited.

\begin{abstract}
Soft set theory is a newly emerging tool to deal with uncertain problems. Based on soft sets, soft rough approximation operators are introduced, and soft rough sets are defined by using soft rough approximation operators. Soft rough sets, which could provide a better approximation than rough sets do, can be seen as a generalized rough set model. This paper is devoted to investigating soft rough approximation operations and relationships among soft sets, soft rough sets, and topologies. We consider four pairs of soft rough approximation operators and give their properties. Four sorts of soft rough sets are investigated, and their related properties are given. We show that Pawlak's rough set model can be viewed as a special case of soft rough sets, obtain the structure of soft rough sets, give the structure of topologies induced by a soft set, and reveal that every topological space on the initial universe is a soft approximating space.
\end{abstract}

\section{Introduction}

Most of traditional methods for formal modeling, reasoning, and computing are crisp, deterministic, and precise in character. However, many practical problems within fields such as economics, engineering, environmental science, medical science, and social sciences involve data that contain uncertainties. We cannot use traditional methods because of various types of uncertainties present in these problems.

There are several theories: probability theory, fuzzy set theory, theory of interval mathematics, and rough set theory [1], which we can consider as mathematical tools for dealing with uncertainties. But all these theories have their own difficulties (see [2]). For example, theory of probabilities can deal only with stochastically stable phenomena. To overcome these kinds of difficulties, Molodtsov [2] proposed a completely new approach, which is called soft set theory, for modeling uncertainty.

Presently, works on soft set theory are progressing rapidly. Maji et al. [3-5] further studied soft set theory, used this theory to solve some decision making problems, and devoted fuzzy soft sets combining soft sets with fuzzy sets. Roy et al.
[6] presented a fuzzy soft set theoretic approach towards decision making problems. Jiang et al. [7] extended soft sets with description logics. Aktaş et al. [8] defined soft groups. Feng et al. $[9,10]$ investigated relationships among soft sets, rough sets, and fuzzy sets. Shabir et al. [11] investigated soft topological spaces. Ge et al. [12] discussed relationships between soft sets and topological spaces.

The purpose of this paper is to investigate soft rough approximation operators and relationships among soft sets, soft rough sets, and topologies.

The remaining part of this paper is organized as follows. In Section 2, we recall some basic concepts of rough sets and soft sets. In Section 3, we consider four pairs of soft rough approximation operators and give their properties. Four sorts of soft rough sets are introduced or investigated, and the fact that Pawlak's rough set model can be viewed as a special case of soft rough sets is proved. In Section 4, we investigate the relationships between soft sets and topologies, obtain the structure of topologies induced by a soft set, and reveal that every topological space on the initial universe is a soft approximating space. In Section 5, we give the related properties of soft rough sets and obtain the structure of soft 
rough sets. In Section 6, we prove that there exists a one-toone correspondence between the set of all soft sets and the set of all formal contexts. Conclusion is in Section 7.

\section{Overview of Rough Sets and Soft Sets}

In this section, we recall some basic concepts about rough sets and soft sets.

Throughout this paper, $U$ denotes initial universe, $E$ denotes the set of all possible parameters, and $2^{U}$ denotes the family of all subsets of $U$. We only consider the case where both $U$ and $E$ are nonempty finite sets.

2.1. Rough Sets. Rough set theory was initiated by [1] for dealing with vagueness and granularity in information systems. This theory handles the approximation of an arbitrary subset of a universe by two definable or observable subsets called lower and upper approximations. It has been successfully applied to machine learning, intelligent systems, inductive reasoning, pattern recognition, mereology, image processing, signal analysis, knowledge discovery, decision analysis, expert systems, and many other fields (see $[1,13]$ ).

Let $R$ be an equivalence relation on $U$. The pair $(U, R)$ is called a Pawlak approximation space. The equivalence relation $R$ is often called an indiscernibility relation. Using the indiscernibility relation $R$, one can define the following two rough approximations:

$$
\begin{gathered}
R_{*}(X)=\left\{x \in U:[x]_{R} \subseteq X\right\}, \\
R^{*}(X)=\left\{x \in U:[x]_{R} \cap X \neq \emptyset\right\} .
\end{gathered}
$$

$R_{*}(X)$ and $R^{*}(X)$ are called the Pawlak lower approximation and the Pawlak upper approximation of $X$, respectively. In general, we refer to $R_{*}$ and $R^{*}$ as Pawlak rough approximation operators and $R_{*}(X)$ and $R^{*}(X)$ as Pawlak rough approximations of $X$.

The Pawlak boundary region of $X$ is defined by the difference between these Pawlak rough approximations; that is, $\operatorname{Bnd}_{R}(X)=R^{*}(X)-R_{*}(X)$. It can easily be seen that $R_{*}(X) \subseteq X \subseteq R^{*}(X)$.

A set is Pawlak rough if its boundary region is not empty; otherwise, the set is crisp. Thus, $X$ is Pawlak rough if $R_{*}(X) \neq R^{*}(X)$.

We may relax equivalence relations so that rough set theory is able to solve more complicated problems in practice. The classical rough set theory based on equivalence relations has been extended to binary relations [14].

Definition 1 (see [14]). Let $R$ be a binary relation on $U$. The pair $(U, R)$ is called an approximation space. Based on the approximation space $(U, R)$, we define a pair of operations $\underline{R}$, $\bar{R}: 2^{U} \rightarrow 2^{U}$ as follows:

$$
\begin{gathered}
\underline{R}(X)=\{x \in U: R(x) \subseteq X\}, \\
\bar{R}(X)=\{x \in U: R(x) \cap X \neq \emptyset\},
\end{gathered}
$$

where $X \in 2^{U}$ and $R(x)=\{y \in U: x R y\}$.
$\underline{R}(X)$ and $\bar{R}(X)$ are called the lower approximation and the upper approximation of $X$, respectively. In general, we refer to $\underline{R}$ and $\bar{R}$ as rough approximation operators and $\underline{R}(X)$ and $\bar{R}(X)$ as rough approximations of $X$.

$X$ is called a definable set if $\underline{R}(X)=\bar{R}(X) ; X$ is called a rough set if $\underline{R}(X) \neq \bar{R}(X)$.

\subsection{Soft Sets}

Definition 2 (see [2]). Let $A$ be a nonempty subset of $E$. A pair $(f, A)$ is called a soft set over $U$, if $f$ is a mapping given by $f: A \rightarrow 2^{U}$. We denote $(f, A)$ by $f_{A}$.

In other words, a soft set over $U$ is a parametrized family of subsets of the universe $U$. For $e \in A, f(e)$ may be considered as the set of $e$-approximate elements of the soft set $f_{A}$.

Definition 3 (see [3]). Let $f_{A}$ and $g_{B}$ be two soft sets over $U$.

(1) $f_{A}$ is called a soft subset of $g_{B}$, if $A \subseteq B$ and $f(e)=$ $g(e)$ for each $e \in A$. We denote it by $f_{A} \widetilde{\subset} g_{B}$.

(2) $f_{A}$ is called a soft super set of $g_{B}$, if $g_{B} \widetilde{C} f_{A}$. We denote it by $f_{A} \widetilde{\supset} g_{B}$.

Definition 4 (see [3]). Let $f_{A}$ and $g_{B}$ be two soft sets over $U$. $f_{A}$ and $g_{B}$ are called soft equal, if $A=B$ and $f(e)=g(e)$ for each $e \in A$. We denote it by $f_{A}=g_{B}$.

Obviously, $f_{A}=g_{B}$ if and only if $f_{A} \tilde{\subset} g_{B}$ and $f_{A} \tilde{\supset} g_{B}$.

Definition 5 (see $[10,12])$. Let $f_{A}$ be a soft set over $U$.

(1) $f_{A}$ is called full, if $\bigcup_{a \in A} f(a)=U$.

(2) $f_{A}$ is called partition, if $\{f(a): a \in A\}$ forms a partition of $U$.

Obviously, every partition soft set is full.

Definition 6. Let $f_{A}$ be a soft set over $U$.

(1) $f_{A}$ is called keeping intersection, if for any $a, b \in A$, there exists $c \in A$ such that $f(a) \cap f(b)=f(c)$.

(2) $f_{A}$ is called keeping union, if for any $a, b \in A$, there exists $c \in A$ such that $f(a) \cup f(b)=f(c)$.

(3) $f_{A}$ is called topological, if $\{f(a): a \in A\}$ is a topology on $U$.

Obviously, every topological soft set is full, keeping intersection and keeping union, and $f_{A}$ is keeping intersection (resp., keeping union) if and only if for any $A^{\prime} \subseteq A$, there exists $a^{\prime} \in A$ such that $\bigcap_{a \in A^{\prime}} f(a)=f\left(a^{\prime}\right)$ (resp., $\left.\bigcup_{a \in A^{\prime}} f(a)=f\left(a^{\prime}\right)\right)$. 
Example 7. Let $U=\left\{h_{1}, h_{2}, h_{3}, h_{4}, h_{5}\right\}, A=\left\{a_{1}, a_{2}, a_{3}, a_{4}\right\}$, and let $f_{A}$ be a soft set over $U$, defined as follows:

$$
\begin{aligned}
& f\left(a_{1}\right)=\left\{h_{1}, h_{2}, h_{5}\right\}, \\
& f\left(a_{2}\right)=\emptyset, \\
& f\left(a_{3}\right)=\left\{h_{3}\right\}, \\
& f\left(a_{4}\right)=\left\{h_{3}, h_{4}\right\} .
\end{aligned}
$$

Obviously, $f_{A}$ is not partition. We have

$$
\begin{aligned}
f\left(a_{3}\right) \cap f\left(a_{4}\right) & =\left\{h_{3}\right\}=f\left(a_{3}\right), \\
f\left(a_{1}\right) \cap f\left(a_{2}\right) & =f\left(a_{1}\right) \cap f\left(a_{3}\right)=f\left(a_{1}\right) \cap f\left(a_{4}\right) \\
& =f\left(a_{2}\right) \cap f\left(a_{3}\right)=f\left(a_{2}\right) \cap f\left(a_{4}\right) \\
& =\emptyset=f\left(a_{2}\right) .
\end{aligned}
$$

Then, $f_{A}$ is full and keeping intersection. But

$$
f\left(a_{1}\right) \cup f\left(a_{3}\right)=\left\{h_{1}, h_{2}, h_{3}, h_{5}\right\} \neq f(a) \quad(\forall a \in A) .
$$

Thus, $f_{A}$ is not keeping union.

Example 8. Let $U=\left\{h_{1}, h_{2}, h_{3}, h_{4}, h_{5}\right\}, A=\left\{a_{1}, a_{2}, a_{3}, a_{4}\right\}$, and let $f_{A}$ be a soft set over $U$, defined as follows:

$$
\begin{aligned}
& f\left(a_{1}\right)=\left\{h_{1}\right\}, \\
& f\left(a_{2}\right)=\left\{h_{1}, h_{2}\right\}, \\
& f\left(a_{3}\right)=\left\{h_{1}, h_{2}, h_{3}\right\}, \\
& f\left(a_{4}\right)=\left\{h_{1}, h_{2}, h_{3}, h_{4}\right\} .
\end{aligned}
$$

Then, $f_{A}$ is keeping intersection and keeping union. But $f_{A}$ is not full.

Example 9. Let $U=\left\{h_{1}, h_{2}, h_{3}, h_{4}, h_{5}\right\}, A=\left\{a_{1}, a_{2}, a_{3}, a_{4}\right\}$, and let $f_{A}$ be a soft set over $U$, defined as follows:

$$
\begin{aligned}
& f\left(a_{1}\right)=\left\{h_{1}\right\}, \\
& f\left(a_{2}\right)=\left\{h_{2}\right\}, \\
& f\left(a_{3}\right)=\left\{h_{1}, h_{2}\right\}, \\
& f\left(a_{4}\right)=U .
\end{aligned}
$$

Then, $f_{A}$ is full and keeping union. But $f_{A}$ is neither keeping intersection nor partition.

Example 10. Let $U=\left\{h_{1}, h_{2}, h_{3}, h_{4}, h_{5}\right\}, A=\left\{a_{1}, a_{2}, a_{3}, a_{4}\right\}$, and let $f_{A}$ be a soft set over $U$, defined as follows:

$$
\begin{aligned}
& f\left(a_{1}\right)=\left\{h_{1}, h_{2}\right\}, \\
& f\left(a_{2}\right)=\left\{h_{5}\right\}, \\
& f\left(a_{3}\right)=\left\{h_{3}\right\}, \\
& f\left(a_{4}\right)=\left\{h_{4}\right\} .
\end{aligned}
$$

Obviously, $f_{A}$ is partition. But

$$
\begin{aligned}
& f\left(a_{2}\right) \cap f\left(a_{3}\right)=\emptyset \neq f(a) \quad(\forall a \in A), \\
& f\left(a_{1}\right) \cup f\left(a_{2}\right)=\left\{h_{1}, h_{2}, h_{5}\right\} \neq f(a) \quad(\forall a \in A) .
\end{aligned}
$$
union.

Thus, $f_{A}$ is neither keeping intersection nor keeping

Example 11. Let $U=\left\{h_{1}, h_{2}, h_{3}, h_{4}, h_{5}\right\}, A=\left\{a_{1}, a_{2}, a_{3}, a_{4}\right\}$ and let $f_{A}$ be a soft set over $U$, defined as follows

$$
\begin{aligned}
& f\left(a_{1}\right)=\left\{h_{1}\right\}, \\
& f\left(a_{2}\right)=\left\{h_{1}, h_{4}\right\}, \\
& f\left(a_{3}\right)=\left\{h_{1}, h_{3}, h_{4}\right\}, \\
& f\left(a_{4}\right)=X .
\end{aligned}
$$

Obviously, $f_{A}$ is full, keeping intersection and keeping union. But $f_{A}$ is not topological.

From Examples 7, 8, 9, and 10 and 11, we have the following relationships:
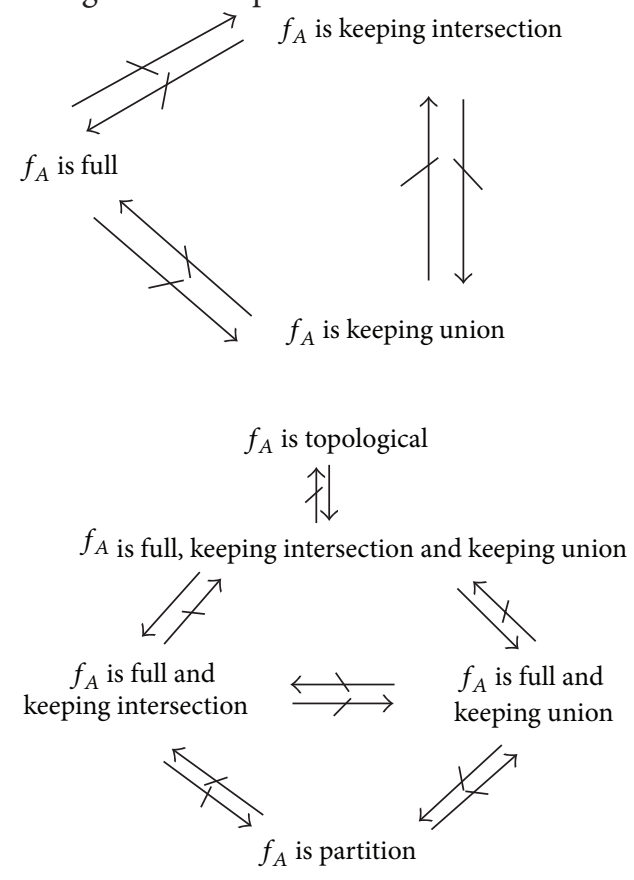

\section{Soft Rough Approximation Operators and Soft Rough Sets}

Soft rough sets, which could provide a better approximation than rough sets do, can be seen as a generalized rough set model (see Example 4.6 in [10]), and defining soft rough sets and some related concepts needs using soft rough approximation operators based on soft sets. Thus, soft rough approximation operators deserve further research.

In this section, we consider a pair of soft rough approximation operators which are presented by Feng et al. in $[9,10]$, proposing three pairs of soft rough approximation operators 
and giving their properties. Four sorts of soft rough sets are defined by using four pairs of soft rough approximation operators.

\subsection{Soft Rough Approximation Operators $\underline{a p r}_{P}$ and $\overline{a p r}_{P}$}

Definition 12 (see $[9,10])$. Let $f_{A}$ be a soft set over $U$. Then, the pair $P=\left(U, f_{A}\right)$ is called a soft approximation space. We define a pair of operators apr ${ }_{P}, \overline{\operatorname{apr}}_{P}: 2^{U} \rightarrow 2^{U}$ as follows:

$$
\begin{gathered}
\underset{\operatorname{apr}_{P}}{ }(X)=\{u \in U: \exists a \in A \text {, s.t. } u \in f(a) \subseteq X\} \\
\overline{\operatorname{apr}}_{P}(X)=\{u \in U: \exists a \in A, \\
\text { s.t. } u \in f(a), f(a) \cap X \neq \emptyset\}
\end{gathered}
$$

$\operatorname{apr}_{P}$ and $\overline{\operatorname{apr}}_{P}$ are called the soft $P$-lower approximation operator on $U$ and the soft $P$-upper approximation operator on $U$, respectively. In general, we refer to $\underline{\operatorname{apr}}_{P}$ and $\overline{\operatorname{apr}}_{P}$ as soft $P$-rough approximations operator on $U$.

$\underline{\operatorname{apr}}_{P}(X)$ and $\overline{\operatorname{apr}}_{P}(X)$ are called the soft $P$-lower approximation and the soft $P$-upper approximation of $X$, respectively. In general, we refer to $\operatorname{apr}_{P}(X)$ and $\overline{\operatorname{apr}}_{P}(X)$ as soft rough approximations of $X$ with respect to $P$.

$X$ is called a soft $P$-definable set if $\operatorname{apr}_{P}(X)=\overline{\operatorname{apr}}_{P}(X) ; X$ is called a soft $P$-rough set if $\operatorname{apr}_{P}(X) \neq \overline{\overline{\operatorname{apr}}_{P}}(X)$.

Moreover, the sets

$$
\begin{gathered}
\operatorname{Pos}_{P}(X)=\underline{\operatorname{apr}_{P}}(X), \\
\operatorname{Neg}_{P}(X)=U-\overline{\operatorname{apr}}_{P}(X), \\
\operatorname{Bnd}_{P}(X)=\overline{\operatorname{apr}}_{P}(X)-\underline{\operatorname{apr}}(X)
\end{gathered}
$$

are called the soft $P$-positive region, the soft $P$-negative region, and the soft $P$-boundary region of $A$, respectively.

Proposition 13 (see $[9,10]$ ). Let $f_{A}$ be a soft set over $U$, and let $P=\left(U, f_{A}\right)$ be a soft approximation space. Then, the following properties hold for any $X, Y \in 2^{U}$.

(1) $\frac{\operatorname{apr}}{X \cdot{ }_{P}}(X)=\bigcup\{f(a): a \in A$ and $f(a) \subseteq X\} \subseteq$ $\overline{X ;} \overline{\operatorname{apr}}_{P}(X)=\bigcup\{f(a): a \in A$ and $f(a) \cap X \neq \emptyset\}$.

(2) $\underline{\operatorname{apr}}_{P}(\emptyset)=\overline{\operatorname{apr}}_{P}(\emptyset)=\emptyset ; \underline{a p r}_{P}(U)=\overline{\operatorname{apr}}_{P}(U)=$ $\bigcup_{a \in A} f(a)$.

(3) $X \subseteq Y \Rightarrow \underline{a p r}_{P}(X) \subseteq \underline{a p r}_{P}(Y) ; X \subseteq Y \Rightarrow \overline{a p r}_{P}(X) \subseteq$ $\overline{a p r}_{P}(Y)$.

(4) $\overline{\operatorname{apr}}_{P}(X \cup Y)=\overline{\operatorname{apr}}_{P}(X) \cup \overline{\operatorname{apr}}_{P}(Y)$.

(5) $\underset{\overline{\operatorname{apr}}_{P}(X) .}{\operatorname{apr}}\left({ }_{P}(X)\right)=\frac{\operatorname{apr}}{P}(X) ; \underline{\operatorname{apr}}_{P}\left(\overline{a p r}_{P}(X)\right)=$

Proposition 14. Let $f_{A}$ be a soft set over $U$, and let $P=\left(U, f_{A}\right)$ be a soft approximation space. Then, the following properties hold.
(1) If $f_{A}$ is full, then
(a) $\underset{\operatorname{apr}}{P}(X) \subseteq X \subseteq \overline{\operatorname{apr}}_{P}(X)$ for any $X \in 2^{U}$;
(b) $\underset{\operatorname{apr}}{P}(U)=\overline{\operatorname{apr}}_{P}(U)=U$.

(2) If $f_{A}$ is keeping union, then

(a) for any $X \in 2^{U}$, there exists $a \in A$ such that $\underset{\operatorname{apr}}{{ }_{P}}(X)=f(a)$;

(b) for any $X \in 2^{U}$, there exists $a \in A$ such that $\overline{\operatorname{apr}}_{P}(X)=f(a)$.

(3) If $f_{A}$ is keeping intersection, then

$\underset{P}{\operatorname{apr}}(X \cap Y)=\underline{\operatorname{apr}}_{P}(X) \cap \underline{\operatorname{apr}}_{P}(Y)$ for any $X, Y \in 2^{U}$.

(4) If $f_{A}$ is partition, then

$\underset{\operatorname{apr}}{P}(X \cap Y)=\underline{\operatorname{apr}}_{P}(X) \cap \underline{\operatorname{apr}}_{P}(Y)$ for any $X, Y \in 2^{U}$.

(5) If $f_{A}$ is full and keeping union, then

$$
\overline{\operatorname{apr}}_{P}(X)=U \text { for any } X \in 2^{U} \backslash\{\emptyset\} .
$$

Proof. (1)(a) By Proposition 13, $\operatorname{apr}_{P}(X) \subseteq X$. Suppose that $X-\overline{\operatorname{apr}}_{P}(X) \neq \emptyset$. Pick

$$
x \in X-\overline{\operatorname{apr}}_{P}(X) \neq \emptyset .
$$

Since $f_{A}$ is full, $U=\bigcup_{a \in A} f(a)$. So, $x \in f(a)$ for some $a \in A$. $x \in X$ implies $f(a) \cap X \neq \emptyset$. Thus, $x \in \overline{\operatorname{apr}}_{P}(X) \neq \emptyset$, contradiction. Hence,

$$
X \subseteq \overline{\operatorname{apr}}_{P}(X) .
$$

(1)(b) This holds by (1) and Proposition 13.

(2) This holds by Proposition 13.

(3) By Proposition 13,

$$
\underline{\operatorname{apr}}_{P}(X \cap Y) \subseteq \underline{\operatorname{apr}}_{P}(X) \cap \underline{\operatorname{apr}}_{P}(Y) .
$$

Suppose that $\underline{\operatorname{apr}}_{P}(X) \cap \underline{\operatorname{apr}}_{P}(Y)-\underline{\operatorname{apr}}_{P}(X \cap Y) \neq \emptyset$. Pick

$$
x \in \underline{\operatorname{apr}}_{P}(X) \cap \underline{\operatorname{apr}}_{P}(Y)-\underline{\operatorname{apr}}_{P}(X \cap Y) .
$$

Then, there exist $a, b \in A$ such that $x \in f(a) \subseteq X$ and $x \in f(b) \subseteq Y$. Since $f_{A}$ is keeping intersection, then $f(a) \cap$ $f(b)=f(c)$ for some $c \in A$. This implies $x \in f(c) \subseteq X \cap Y$. Thus, $x \in \underline{\operatorname{apr}}_{P}(X \cap Y)$, contradiction. Hence,

$$
\underline{\operatorname{apr}}_{P}(X \cap Y) \supseteq \underline{\operatorname{apr}}_{P}(X) \cap \underline{\operatorname{apr}}_{P}(Y) .
$$

Therefore,

$$
\underline{\operatorname{apr}}_{P}(X \cap Y)=\underline{\operatorname{apr}}_{P}(X) \cap \underline{\operatorname{apr}}_{P}(Y) .
$$


(4) Suppose that $\underline{\operatorname{apr}}_{P}(X) \cap \underline{\operatorname{apr}}_{P}(Y)-\underline{\operatorname{apr}}_{P}(X \cap Y) \neq \emptyset$. Pick

$$
x \in \underline{\operatorname{apr}}_{P}(X) \cap \underline{\operatorname{apr}}_{P}(Y)-\underline{\operatorname{apr}}_{P}(X \cap Y) .
$$

Then, there exist $a, b \in A$ such that $x \in f(a) \subseteq X$ and $x \in f(b) \subseteq Y$. Since $f_{A}$ is partition, then $f(a)=f(b)$. This implies $x \in f(a) \subseteq X \cap Y$. So, $x \in \operatorname{apr}_{P}(X \cap Y)$, contradiction. Thus,

$$
\underline{\operatorname{apr}}_{P}(X \cap Y) \supseteq \underline{\operatorname{apr}}_{P}(X) \cap \underline{\operatorname{apr}}_{P}(Y) .
$$

Therefore,

$$
\underline{\operatorname{apr}}_{P}(X \cap Y)=\underline{\operatorname{apr}}_{P}(X) \cap \underline{\operatorname{apr}}_{P}(Y) .
$$

(5) Since $f_{A}$ is full and keeping union, then $U=$ $\bigcup_{a \in A} f(a)=f\left(a^{*}\right)$ for some $a^{*} \in A$. For each $X \in 2^{U} \backslash\{\emptyset\}$ and each $u \in U, u \in f\left(a^{*}\right)$ and $f\left(a^{*}\right) \cap X=X \neq \emptyset$, and then $\overline{\operatorname{apr}}_{P}(X)=U$.

3.2. Soft Rough Approximation Operators $\underline{a p r}_{P}^{\prime}$ and $\overline{\operatorname{apr}}_{P}^{\prime}$, $\underline{\operatorname{apr}}_{P}^{\prime \prime}$ and $\overline{\operatorname{apr}}_{P}^{\prime \prime}$, and $\underline{\operatorname{apr}}_{P}^{\prime \prime \prime}$ and $\overline{\operatorname{apr}}_{P}^{\prime \prime \prime}$

Definition 15. Let $f_{A}$ be a soft set over $U$.

(1) Define a binary relation $R_{f}$ on $U$ by

$$
x R_{f} y \Longleftrightarrow \exists a \in A, \quad\{x, y\} \subseteq f(a)
$$

for each $x, y \in U$. Then, $R_{f}$ is called the binary relation induced by $f_{A}$ on $U$.

(2) For each $x \in U$, define a successor neighborhood $R_{f_{s}}(x)$ of $x$ in $U$ by

$$
\left(R_{f}\right)_{s}(x)=\left\{y \in U: x R_{f} y\right\} .
$$

Since the following Proposition 16 is clear, we omit its proof.

Proposition 16. Let $f_{A}$ be a soft set over $U$, and let $R_{f}$ be the binary relation induced by $f_{A}$ on $U$. Then, the following properties hold.

(1) $R_{f}$ is a symmetric relation.

(2) If $f_{A}$ is full, then $R_{f}$ is a reflexive relation.

(3) If $f_{A}$ is partition, then $R_{f}$ is an equivalence relation.

Proposition 17. Let $f_{A}$ be a soft set over $U$, and let $R_{f}$ be the binary relation induced by $f_{A}$ on $U$. Then, the following properties hold.

(1) If $x \in f(a)$ with $a \in A$, then $f(a) \subseteq\left(R_{f}\right)_{s}(x)$.

(2) If $f_{A}$ is partition and $x \in f(a)$ with $a \in A$, then $f(a)=$ $\left(R_{f}\right)_{s}(x)$.

(3) If $f_{A}$ is keeping union, then for each $x \in U$, there exists $a \in A$ such that $\left(R_{f}\right)_{s}(x)=f(a)$.
Proof. (1) This is obvious.

(2) Suppose that $y \in\left(R_{f}\right)_{s}(x)$. Then, $x R_{f} y$, and so $\{x, y\} \subseteq f(b)$ for some $b \in A$. Since $f_{A}$ is partition and $f(a) \cap f(b) \neq \emptyset$, then $f(a)=f(b)$. Thus, $y \in f(a)$. This implies $f(a) \supseteq\left(R_{f}\right)_{s}(x)$. By $(1)$,

$$
f(a)=\left(R_{f}\right)_{s}(x) .
$$

(3) Suppose that $y \in\left(R_{f}\right)_{s}(x)$. Then, $x R_{f} y$, and so $\{x, y\} \subseteq f\left(a_{y}\right)$ for some $a_{y} \in A$. By $(1), f\left(a_{y}\right) \subseteq\left(R_{f}\right)_{s}(x)$. Thus, $\{y\} \subseteq f\left(a_{y}\right) \subseteq\left(R_{f}\right)_{s}(x)$. This implies

$$
\left(R_{f}\right)_{s}(x)=\bigcup\left\{f\left(a_{y}\right): y \in\left(R_{f}\right)_{s}(x)\right\} .
$$

Since $f_{A}$ is keeping union, then $\bigcup\left\{f\left(a_{y}\right): y \in\right.$ $\left.\left(R_{f}\right)_{s}(x)\right\}=f(a)$ for some $a \in A$. Thus,

$$
\left(R_{f}\right)_{s}(x)=f(a) .
$$

Definition 18. Let $f_{A}$ be a soft set over $U$, and let $P=\left(U, f_{A}\right)$ be a soft approximation space. We define three pairs of soft rough approximation operations: $2^{U} \rightarrow 2^{U}$ as follows:

(1)

$$
\begin{gathered}
\operatorname{apr}_{P}^{\prime}(X)=\left\{x \in U:\left(R_{f}\right)_{s}(x) \subseteq X\right\}, \\
\overline{\operatorname{apr}}_{P}^{\prime}(X)=\left\{x \in U:\left(R_{f}\right)_{s}(x) \cap X \neq \emptyset\right\} .
\end{gathered}
$$

$X$ is called a soft $P^{\prime}$-definable set if $\operatorname{apr}_{P}(X)=\overline{\operatorname{apr}}_{P}^{\prime}(X) . X$ is called a soft $P^{\prime}$-rough set if $\underline{\operatorname{apr}}_{P}^{\prime}(X) \neq \overline{\operatorname{apr}}_{P}^{\prime}(X)$. The sets

$$
\begin{gathered}
\operatorname{Pos}_{P}^{\prime}(X)=\underline{\operatorname{apr}}_{P}^{\prime}(X), \\
\operatorname{Neg}_{P}^{\prime}(X)=U-\overline{\operatorname{apr}}_{P}^{\prime}(X), \\
\operatorname{Bnd}_{P}^{\prime}(X)=\overline{\operatorname{apr}}_{P}^{\prime}(X)-\underline{\operatorname{apr}}_{P}^{\prime}(X)
\end{gathered}
$$

are called the soft $P^{\prime}$-positive region, the soft $P^{\prime}$-negative region, and the soft $P^{\prime}$-boundary region of $X$, respectively. Consider,

(2)

$$
\begin{aligned}
\underline{\operatorname{apr}}_{P}^{\prime \prime}(X)= & \bigcup\left\{\left(R_{f}\right)_{s}(x): x \in U,\left(R_{f}\right)_{s}(x) \subseteq X\right\}, \\
& \overline{\operatorname{apr}}_{P}^{\prime \prime}(X)=U-\underline{\operatorname{apr}_{P}^{\prime \prime}}(U-X) .
\end{aligned}
$$

$X$ is called a soft $P^{\prime \prime}$-definable set if $\operatorname{apr}_{P}^{\prime \prime}(X)=\overline{\operatorname{apr}}_{P}^{\prime \prime}(X)$. $X$ is called a soft $P^{\prime \prime}$-rough set if $\underline{\operatorname{apr}}_{P}^{\prime \prime}(X) \neq \overline{\operatorname{apr}}_{P}^{\prime \prime}(X)$. The sets

$$
\begin{gathered}
\operatorname{Pos}_{P}^{\prime \prime}(X)=\underline{\operatorname{apr}}_{P}^{\prime \prime}(X), \\
\operatorname{Neg}_{P}^{\prime \prime}(X)=U-\overline{\operatorname{apr}}_{P}^{\prime \prime}(X), \\
\operatorname{Bnd}_{P}^{\prime \prime}(X)=\overline{\operatorname{apr}}_{P}^{\prime \prime}(X)-\underline{\operatorname{apr}}_{P}^{\prime \prime}(X)
\end{gathered}
$$

are called the soft $P^{\prime \prime}$-positive region, the soft $P^{\prime \prime}$-negative region, and the soft $P^{\prime \prime}$-boundary region of $X$, respectively. Consider, 
(3)

$$
\begin{aligned}
\overline{\operatorname{apr}}_{P}^{\prime \prime \prime}(X)= & \cup\left\{\left(R_{f}\right)_{s}(x): x \in U,\left(R_{f}\right)_{s}(x) \cap X \neq \emptyset\right\}, \\
& \underline{\operatorname{apr}}_{P}^{\prime \prime \prime}(X)=U-\overline{\operatorname{apr}}_{P}^{\prime \prime \prime}(U-X) .
\end{aligned}
$$

$X$ is called a soft $P^{\prime \prime \prime}$-definable set if $\underline{\operatorname{apr}}_{P}^{\prime \prime \prime}(X)=\overline{\operatorname{apr}}_{P}^{\prime \prime \prime}(X)$. $X$ is called a soft $P^{\prime \prime \prime}$-rough set if $\underline{\operatorname{apr}}_{P}^{\prime \prime \prime}(X) \neq \overline{\operatorname{apr}}_{P}^{\prime \prime \prime}(X)$. The sets

$$
\begin{gathered}
\operatorname{Pos}_{P}^{\prime \prime \prime}(X)=\underline{\operatorname{apr}}_{P}^{\prime \prime \prime}(X), \\
\operatorname{Neg}_{P}^{\prime \prime \prime}(X)=U-\overline{\operatorname{apr}}_{P}^{\prime \prime \prime}(X), \\
\operatorname{Bnd}_{P}^{\prime \prime \prime}(X)=\overline{\operatorname{apr}}_{P}^{\prime \prime \prime}(X)-\underline{\operatorname{apr}_{P}^{\prime \prime \prime}}(X)
\end{gathered}
$$

are called the soft $P^{\prime \prime \prime}$-positive region, the soft $P^{\prime \prime \prime}$-negative region, and the soft $P^{\prime \prime \prime}$-boundary region of $X$, respectively.

In general, we also refer to $\underline{\operatorname{apr}}_{P}^{\prime}(X)$ and $\overline{\operatorname{apr}}_{P}^{\prime}(X), \underline{\operatorname{apr}}_{P}^{\prime \prime}(X)$ and $\overline{\operatorname{apr}}_{P}^{\prime \prime}(X)$, and $\operatorname{apr}_{P}^{\prime \prime \prime}(X)$ and $\overline{\operatorname{apr}}_{P}^{\prime \prime \prime}(X)$ as soft rough approximations of $X$ with respect to $P^{\prime}, P^{\prime \prime}, P^{\prime \prime \prime}$, respectively.

It is not very difficult to prove Propositions 19, 20, and 21 (see [15]).

Proposition 19. Let $f_{A}$ be a soft set over $U$, and let $P=\left(U, f_{A}\right)$ be a soft approximation space. Then, the following properties hold for any $X, Y \in 2^{U}$.

(1) $\operatorname{apr}_{P}^{\prime}(X) \subseteq X$. If $f_{A}$ is full, then

$$
\underline{\operatorname{apr}}_{P}^{\prime}(X) \subseteq X \subseteq \overline{\operatorname{apr}}_{P}^{\prime}(X) .
$$

(2) $\overline{\operatorname{apr}}_{P}^{\prime}(\emptyset)=\emptyset ; \underline{a p r}_{P}^{\prime}(U)=U$. If $f_{A}$ is full, then

$$
\begin{aligned}
& \underline{a p r}_{P}^{\prime}(\emptyset)=\overline{\operatorname{apr}}_{P}^{\prime}(\emptyset)=\emptyset ; \\
& \underline{a p r}_{P}^{\prime}(U)=\overline{\operatorname{apr}}_{P}^{\prime}(U)=U .
\end{aligned}
$$

(3) $X \subseteq Y \Rightarrow \underline{a p r}_{P}^{\prime}(X) \subseteq \underline{a p r}_{P}^{\prime}(Y) ; X \subseteq Y \Rightarrow \overline{\operatorname{apr}}_{P}^{\prime}(X) \subseteq$ $\overline{\operatorname{apr}}_{P}^{\prime}(Y)$.

(4) $\underline{\operatorname{apr}}_{P}^{\prime}(X \cap Y)=\underline{\operatorname{apr}} \underline{P}_{P}^{\prime}(X) \cap \underline{\operatorname{apr}} \underline{P}_{P}^{\prime}(Y)$.

(5) $\overline{\operatorname{apr}}_{P}^{\prime}(X \cup Y)=\overline{\operatorname{apr}}_{P}^{\prime}(X) \cup \overline{\operatorname{apr}}_{P}^{\prime}(Y)$.

(6) $\underline{a p r}_{P}^{\prime}(U-X)=U-\overline{\operatorname{apr}}_{P}^{\prime}(X) ; \overline{\operatorname{apr}}_{P}^{\prime}(U-X)=U-$ $\overline{a p r}_{P}^{\prime}(X)$.

(7) $\left.\overline{\operatorname{apr}}_{P}^{\prime} \underline{\operatorname{apr}}_{P}^{\prime}(X)\right) \subseteq X \subseteq \underline{\operatorname{apr}} \underline{P}_{P}^{\prime}\left(\overline{\operatorname{apr}}_{P}^{\prime}(X)\right)$.

Proposition 20. Let $f_{A}$ be a soft set over $U$, and let $P=$ $\left(U, f_{A}\right)$ be a soft approximation space. Then, the following properties hold for any $X \in 2^{U}$.

(1)

$$
\underline{\operatorname{apr}}_{P}^{\prime \prime}(X) \subseteq X \subseteq \overline{\operatorname{apr}}_{P}^{\prime \prime}(X) .
$$

(2) $\underline{\operatorname{apr}}_{P}^{\prime \prime}(\emptyset)=\emptyset ; \overline{\operatorname{apr}}_{P}^{\prime \prime}(U)=U$. If $f_{A}$ is full, then

$$
\begin{aligned}
& \underline{\operatorname{apr}}_{P}^{\prime}(\emptyset)=\overline{\operatorname{apr}}_{P}^{\prime}(\emptyset)=\emptyset ; \\
& \underline{\operatorname{apr}}_{P}^{\prime}(U)=\overline{\operatorname{apr}}_{P}^{\prime}(U)=U .
\end{aligned}
$$

(3) $\operatorname{apr}_{P}^{\prime \prime}(U-X)=U-\overline{\operatorname{apr}}_{P}^{\prime \prime}(X) ; \overline{\operatorname{apr}}_{P}^{\prime \prime}(U-X)=U-$ $\operatorname{apr}_{P}^{\prime \prime}(X)$.

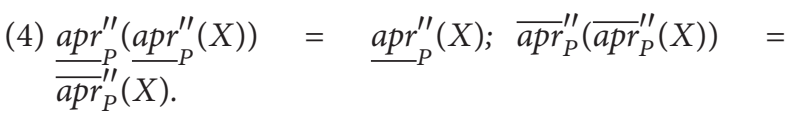

Proposition 21. Let $f_{A}$ be a soft set over $U$, and let $P=\left(U, f_{A}\right)$ be a soft approximation space. Then, the following properties hold for any $X, Y \in 2^{U}$.

(1) If $f_{A}$ is full, then

$$
\underline{a p r}_{P}^{\prime \prime \prime}(X) \subseteq X \subseteq \overline{\operatorname{apr}}_{P}^{\prime \prime \prime}(X)
$$

(2) $\overline{\operatorname{apr}}_{P}^{\prime \prime \prime}(\emptyset)=\emptyset ; \underline{\operatorname{apr}}_{P}^{\prime \prime \prime}(U)=U$. If $f_{A}$ is full, then

$$
\begin{aligned}
& \underline{\operatorname{apr}}_{P}^{\prime \prime \prime}(\emptyset)=\overline{\operatorname{apr}}_{P}^{\prime \prime \prime}(\emptyset)=\emptyset ; \\
& \underline{\operatorname{apr}}_{P}^{\prime \prime \prime}(U)=\overline{\operatorname{apr}}_{P}^{\prime}(U)=U .
\end{aligned}
$$

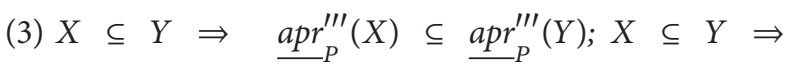
$\overline{\operatorname{apr}}_{P}^{\prime \prime \prime}(X) \subseteq \overline{\operatorname{apr}}_{P}^{\prime \prime \prime}(Y)$.

(4) $\underline{\operatorname{apr}}_{P}^{\prime \prime \prime}(X \cap Y)=\underline{\operatorname{apr}} \underline{P}_{P}^{\prime \prime \prime}(X) \cap \underline{\operatorname{apr}}_{P}^{\prime \prime \prime}(Y)$.

(5) $\overline{\operatorname{apr}}_{P}^{\prime \prime \prime}(X \cup Y)=\overline{\operatorname{apr}}_{P}^{\prime \prime \prime}(X) \cup \overline{\operatorname{apr}}_{P}^{\prime \prime \prime}(Y)$.

(6) $\operatorname{apr}_{P}^{\prime \prime \prime}(U-X)=U-\overline{\operatorname{apr}}_{P}^{\prime \prime \prime}(X) ; \overline{\operatorname{apr}}_{P}^{\prime \prime \prime}(U-X)=U-$ $\underline{\operatorname{apr}}_{P}^{\prime \prime \prime}(X)$.

(7) $\underline{\operatorname{apr}}_{P}^{\prime \prime \prime}\left(\underline{\operatorname{apr}}_{P}^{\prime \prime \prime}(X)\right) \subseteq \quad \underline{\operatorname{apr}}_{P}^{\prime \prime \prime}(X) ; \overline{\operatorname{apr}}_{P}^{\prime \prime \prime}\left(\overline{\operatorname{apr}}_{P}^{\prime \prime \prime}(X)\right) \supseteq$ $\overline{\operatorname{apr}}_{P}^{\prime \prime \prime}(X)$.

(8) $\overline{\operatorname{apr}}_{P}^{\prime \prime \prime}\left(\underline{a p r}_{P}^{\prime \prime \prime}(X)\right) \subseteq X \subseteq \underline{\operatorname{apr}}_{P}^{\prime \prime \prime}\left(\overline{\operatorname{apr}}_{P}^{\prime \prime \prime}(X)\right)$.

Example 22. Let $U=\left\{h_{1}, h_{2}, h_{3}, h_{4}, h_{5}\right\}, A=\left\{a_{1}, a_{2}, a_{3}, a_{4}\right\}$, and let $f_{A}$ be a soft set over $U$, defined as follows:

$$
\begin{aligned}
& f\left(a_{1}\right)=\left\{h_{2}\right\}, \\
& f\left(a_{2}\right)=\left\{h_{1}, h_{4}\right\}, \\
& f\left(a_{3}\right)=\left\{h_{3}\right\}, \\
& f\left(a_{4}\right)=\left\{h_{1}, h_{3}\right\} .
\end{aligned}
$$


Obviously, $f_{A}$ is not full. We have

$$
\begin{aligned}
& \left(R_{f}\right)_{s}\left(h_{1}\right)=\left\{h_{1}, h_{3}, h_{4}\right\}, \\
& \left(R_{f}\right)_{s}\left(h_{2}\right)=\left\{h_{2}\right\}, \\
& \left(R_{f}\right)_{s}\left(h_{3}\right)=\left\{h_{1}, h_{3}\right\}, \\
& \left(R_{f}\right)_{s}\left(h_{4}\right)=\left\{h_{1}, h_{4}\right\}, \\
& \left(R_{f}\right)_{s}\left(h_{5}\right)=\emptyset .
\end{aligned}
$$

Let $X=\left\{h_{3}, h_{5}\right\}, Y=\left\{h_{1}, h_{2}, h_{3}\right\}$, and $Z=\left\{h_{2}, h_{4}, h_{5}\right\}$.

(1) We have

$$
\overline{\operatorname{apr}}_{P}^{\prime}(X)=\left\{h_{1}, h_{3}\right\} .
$$

Thus,

$$
X \nsubseteq \overline{\operatorname{apr}}_{P}^{\prime}(X)
$$

(2) We have

$$
\begin{aligned}
& \underline{\operatorname{apr}}_{P}^{\prime}(X)=\left\{h_{5}\right\}, \\
& \underline{\operatorname{apr}_{P}^{\prime}(Y)=\left\{h_{2}, h_{3}, h_{5}\right\} .}
\end{aligned}
$$

Thus,

$$
\underline{\operatorname{apr}}_{P}^{\prime}(X) \subseteq \underline{\operatorname{apr}}_{P}^{\prime}(Y) \nRightarrow X \subseteq Y .
$$

(3) We have

$$
\begin{aligned}
& \overline{\operatorname{apr}}_{P}^{\prime}(X)=\left\{h_{1}, h_{3}\right\}, \\
& \overline{\operatorname{apr}}_{P}^{\prime}(Y)=\left\{h_{1}, h_{2}, h_{3}, h_{4}\right\} .
\end{aligned}
$$

Thus,

$$
\overline{\operatorname{apr}}_{P}^{\prime}(X) \subseteq \overline{\operatorname{apr}}_{P}^{\prime}(Y) \nRightarrow X \subseteq Y .
$$

(4) We have

$$
\begin{aligned}
& \underline{\operatorname{apr}}_{P}^{\prime}(Y)=\left\{h_{2}, h_{3}, h_{5}\right\}, \\
& \underline{\operatorname{apr}}_{P}^{\prime}(Z)=\left\{h_{2}, h_{5}\right\}, \\
& \underline{\operatorname{apr}}_{P}^{\prime}(Y \cup Z)=U .
\end{aligned}
$$

Thus,

$$
\underline{\operatorname{apr}}_{P}^{\prime}(Y \cup Z) \neq \underline{\operatorname{apr}}_{P}^{\prime}(y) \cup \underline{\operatorname{apr}}_{P}^{\prime}(Z) .
$$

(5) We have

$$
\begin{aligned}
& \overline{\operatorname{apr}}_{P}^{\prime}(Y)=\left\{h_{1}, h_{2}, h_{3}, h_{4}\right\}, \\
& \overline{\operatorname{apr}}_{P}^{\prime}(Z)=\left\{h_{1}, h_{2}, h_{4}\right\}, \\
& \overline{\operatorname{apr}}_{P}^{\prime}(Y \cap Z)=\left\{h_{2}\right\} .
\end{aligned}
$$

Thus,

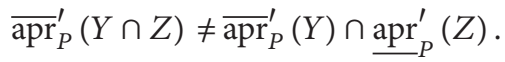

3.3. The Relationships among Four Pairs of Soft Rough Approximation Operators

Lemma 23. Let $f_{A}$ be a soft set over $U$, and let $P=\left(U, f_{A}\right)$ be a soft approximation space. Then, the following properties hold for any $X \in 2^{U}$.

(1) If $f_{A}$ is full, then

$$
\underset{P}{\operatorname{apr}}(X) \supseteq \underline{\operatorname{apr}}_{P}^{\prime}(X) \text {. }
$$

(2) If $f_{A}$ is full and keeping union, then

$$
\overline{\operatorname{apr}}_{P}(X) \supseteq \overline{\operatorname{apr}}_{P}^{\prime}(X) \text {. }
$$

(3) If $f_{A}$ is partition, then
(a) $\operatorname{apr}_{P}(X)=\operatorname{apr}_{p}^{\prime}(X)$
(b) $\overline{\operatorname{apr}}_{P}(X)=\overline{\operatorname{apr}}_{P}^{\prime}(X)$.

Proof. (1) Suppose that $x \in \operatorname{apr}_{p}^{\prime}(X)$. Then, $\left(R_{f}\right)_{s}(x) \subseteq$

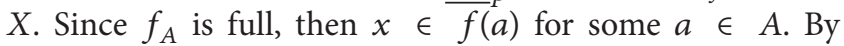
Proposition 17, $f(a) \subseteq\left(R_{f}\right)_{s}(x)$. Thus, $x \in f(a) \subseteq X$. This implies $x \in \operatorname{apr}_{p}(X)$. Thus,

$$
\underline{\operatorname{apr}}_{P}(X) \supseteq \underline{\operatorname{apr}}_{P}^{\prime}(X) .
$$

(2) If $X=\emptyset$, then $\overline{\operatorname{apr}}_{P}(X)=\emptyset=\overline{\operatorname{apr}}_{P}^{\prime}(X)$. If $X \neq \emptyset$, by Proposition 14, $\overline{\operatorname{apr}}_{P}(X)=U$.

Hence,

$$
\overline{\operatorname{apr}}_{P}(X) \supseteq \overline{\operatorname{apr}}_{P}^{\prime}(X) .
$$

(3)(a) Suppose that $x \in \operatorname{apr}_{P}(X)$. Then, $x \in f(a) \subseteq X$ for some $a \in A$. Since $f_{A}$ is partition and $x \in f(a)$, then $f(a)=\left(R_{f}\right)_{s}(x)$ by Proposition 17. This implies $x \in \operatorname{apr}_{P}^{\prime}(X)$. Thus,

$$
\underline{\operatorname{apr}}_{P}(X) \subseteq \underline{\operatorname{apr}}_{P}^{\prime}(X) .
$$

By (1),

$$
\underline{\operatorname{apr}}_{P}(X)=\underline{\operatorname{apr}}_{P}^{\prime}(X) \text {. }
$$

(3)(b) This is similar to the proof of (3) (a).

Suppose that $x \in \overline{\operatorname{apr}}_{P}^{\prime}(X)$. Then, $\left(R_{f}\right)_{s}(x) \cap X \neq \emptyset$. Since $f_{A}$ is full, then $x \in f(a)$ for some $a \in A$. Since $f_{A}$ is partition and $x \in f(a)$, then $f(a)=\left(R_{f}\right)_{s}(x)$ by Proposition 17. This implies $x \in \overline{\operatorname{apr}}_{P}(X)$. Thus,

$$
\overline{\operatorname{apr}}_{P}(X) \supseteq \overline{\operatorname{apr}}_{P}^{\prime}(X) .
$$

Hence, $\overline{\operatorname{apr}}_{P}(X)=\overline{\operatorname{apr}}_{P}^{\prime}(X)$.

By Propositions 13 and 17, we have Lemma 24

Lemma 24. Let $f_{A}$ be a soft set over $U$, and let $P=\left(U, f_{A}\right)$ be a soft approximation space. If $f_{A}$ is keeping union, then for any $X \in 2^{U}$,

$$
\underline{a p r}_{P}(X) \supseteq \underline{a p r}_{P}^{\prime \prime}(X), \quad \overline{a p r}_{P}(X) \supseteq \overline{a p r}_{P}^{\prime \prime \prime}(X) .
$$


Lemma 25. Let $f_{A}$ be a soft set over $U$, and let $P=\left(U, f_{A}\right)$ be a soft approximation space. Then, the following properties hold for any $X \in 2^{U}$.

(1) If $f_{A}$ is full, then

$$
\begin{aligned}
& \underline{a p r}_{P}^{\prime \prime \prime}(X) \subseteq \underline{a p r_{P}^{\prime}}(X) \subseteq \underline{a p r}_{P}^{\prime \prime}(X) \subseteq X \\
& \subseteq \overline{\operatorname{apr}}_{P}^{\prime \prime}(X) \subseteq \overline{\operatorname{apr}}_{P}^{\prime}(X) \subseteq \overline{\operatorname{apr}}_{P}^{\prime \prime \prime}(X) .
\end{aligned}
$$

(2) If $f_{A}$ is partition, then

$$
\begin{aligned}
& \text { (a) }{ }_{\operatorname{apr}}^{\prime}(X)=\underline{a p r}_{P}^{\prime \prime}(X)=\underline{a p r}_{P}^{\prime \prime \prime}(X) ; \\
& \text { (b) } \overline{\operatorname{apr}}_{P}^{\prime}=\overline{\operatorname{apr}}_{P}^{\prime \prime}(X)=\overline{\operatorname{apr}}_{P}^{\prime \prime \prime}(X) .
\end{aligned}
$$

Proof. (1) Suppose that $x \in \operatorname{apr}_{p}^{\prime}(X)$. Then, $\left(R_{f}\right)_{s}(x) \subseteq X$. Since $f_{A}$ is full, then $x \in\left(R_{f}\right)_{s}(x) \subseteq X$ by Proposition 17 . This implies $x \in \operatorname{apr}_{P}^{\prime \prime}(X)$. Thus,

$$
\underline{\operatorname{apr}}_{P}^{\prime}(X) \subseteq \underline{\operatorname{apr}}_{P}^{\prime \prime}(X) .
$$

Suppose that $\underline{\operatorname{apr}}_{P}^{\prime \prime \prime}(X)-\underline{\operatorname{apr}}_{P}^{\prime}(X) \neq \emptyset$. Pick

$$
x \in \underline{\operatorname{apr}}_{P}^{\prime \prime \prime}(X)-\underline{\operatorname{apr}}_{P}^{\prime}(X) .
$$

$x \notin \operatorname{apr}_{P}^{\prime}(X)$ implies $\left(R_{f}\right)_{s}(x) \nsubseteq X$. So, $\left(R_{f}\right)_{s}(x) \cap(U-$ $X) \neq \emptyset$. Since $f_{A}$ is full, then $x \in\left(R_{f}\right)_{s}(x)$ by Proposition 17 . This implies $x \in \overline{\operatorname{apr}}_{P}^{\prime \prime}(U-X)$. Thus, $x \notin U-\overline{\operatorname{apr}}_{P}^{\prime \prime}(U-X)=$ $\operatorname{apr}_{p}^{\prime \prime \prime}(X)$, contradiction.

Hence, $\operatorname{apr}_{p}^{\prime \prime \prime}(X) \subseteq \operatorname{apr}_{p}^{\prime}(X)$.

By Proposition 20,

$$
\underline{\operatorname{apr}}_{P}^{\prime \prime}(X) \subseteq X \subseteq \overline{\operatorname{apr}}_{P}^{\prime \prime}(X) .
$$

Since

$$
\underline{\operatorname{apr}}_{P}^{\prime \prime \prime}(U-X) \subseteq \underline{\operatorname{apr}}_{P}^{\prime}(U-X) \subseteq \underline{\operatorname{apr}}_{P}^{\prime \prime}(U-X),
$$

then

$$
U-\underline{\operatorname{apr}}_{P}^{\prime \prime \prime}(U-X) \supseteq U-\underline{\operatorname{apr}}_{P}^{\prime}(U-X) \supseteq U-\underline{\operatorname{apr}}_{P}^{\prime \prime}(U-X) \text {. }
$$

By Propositions 19, 20, and 21,

$$
\overline{\operatorname{apr}}_{P}^{\prime \prime}(X) \subseteq \overline{\operatorname{apr}}_{P}^{\prime}(X) \subseteq \overline{\operatorname{apr}}_{P}^{\prime \prime \prime}(X) .
$$

(2)(a) Suppose that $x \in \operatorname{apr}_{P}^{\prime \prime}(X)$. Then, there exists $y \in U$ such that $x \in\left(R_{f}\right)_{s}(y) \subseteq X$. Since $f_{A}$ is partition, then $R_{f}$ is an equivalence relation by Proposition 16. Thus, $x \in\left(R_{f}\right)_{s}(y)$ follows $\left(R_{f}\right)_{s}(x)=\left(R_{f}\right)_{s}(y)$. So, $\left(R_{f}\right)_{s}(x) \subseteq X$. This implies $x \in \operatorname{apr}_{p}^{\prime}(X)$. Hence,

$$
\underline{\operatorname{apr}}_{P}^{\prime}(X) \supseteq \underline{\operatorname{apr}}_{P}^{\prime \prime}(X) .
$$

By (1),

$$
\underline{\operatorname{apr}}_{P}^{\prime}(X)=\underline{\operatorname{apr}_{P}^{\prime \prime}}(X)
$$

Suppose that $\underline{\operatorname{apr}}_{P}^{\prime}(X) \cap \overline{\operatorname{apr}}_{P}^{\prime \prime \prime}(U-X) \neq \emptyset$. Pick

$$
x \in \underline{\operatorname{apr}}_{P}^{\prime}(X) \cap \overline{\operatorname{apr}}_{P}^{\prime \prime \prime}(U-X) .
$$

$x \in \underline{\operatorname{apr}}_{P}^{\prime}(X)$ implies $\left(R_{f}\right)_{s}(x) \subseteq X . x \in \overline{\operatorname{apr}}_{P}^{\prime \prime \prime}(U-X)$ implies that there exists $y \in U$ such that $x \in\left(R_{f}\right)_{s}(y)$ and $\left(R_{f}\right)_{s}(y) \cap(U-X) \neq \emptyset$. So, $\left(R_{f}\right)_{s}(y) \nsubseteq X$. Note that $R_{f}$ is an equivalence relation. Then, $\left(R_{f}\right)_{s}(x)=\left(R_{f}\right)_{s}(y)$. Thus, $\left(R_{f}\right)_{s}(x) \nsubseteq X$, contradiction.

Hence, $\operatorname{apr}_{P}^{\prime}(X) \cap \overline{\operatorname{apr}}_{P}^{\prime \prime \prime}(U-X)=\emptyset$.

This proves that

$$
\underline{\operatorname{apr}}_{P}^{\prime}(X) \subseteq U-\overline{\operatorname{apr}}_{P}^{\prime \prime \prime}(U-X)=\underline{\operatorname{apr}}_{P}^{\prime \prime \prime}(X) .
$$

By (1),

$$
\underline{\operatorname{apr}}_{P}^{\prime}(X)=\underline{\operatorname{apr}}_{P}^{\prime \prime \prime}(X) .
$$

(2)(b) By (2)(a),

$$
\underline{\operatorname{apr}}_{P}^{\prime}(U-X)=\underline{\operatorname{apr}}_{P}^{\prime \prime}(U-X)=\underline{\operatorname{apr}}_{P}^{\prime \prime \prime}(U-X) .
$$

Then,

$U-\underline{\operatorname{apr}_{P}^{\prime}}(U-X)=U-\underline{\operatorname{apr}}_{P}^{\prime \prime}(U-X)=U-\underline{\operatorname{apr}}_{P}^{\prime \prime \prime}(U-X)$.

By Propositions 19, 20, and 21,

$$
\overline{\operatorname{apr}}_{P}^{\prime}(X)=\overline{\operatorname{apr}}_{P}^{\prime \prime}(X)=\overline{\operatorname{apr}}_{P}^{\prime \prime \prime}(X) .
$$

Example 26. Let $U=\left\{h_{1}, h_{2}, h_{3}, h_{4}, h_{5}, h_{6}\right\}, A=\left\{a_{1}, a_{2}, a_{3}\right.$, $\left.a_{4}, a_{5}\right\}$, and let $f_{A}$ be a soft set over $U$, defined as follows:

$$
\begin{aligned}
& f\left(a_{1}\right)=\left\{h_{1}\right\}, \\
& f\left(a_{2}\right)=\left\{h_{6}\right\}, \\
& f\left(a_{3}\right)=\left\{h_{2}, h_{5}\right\}, \\
& f\left(a_{4}\right)=\left\{h_{2}, h_{4}\right\}, \\
& f\left(a_{5}\right)=\left\{h_{1}, h_{3}, h_{5}\right\} .
\end{aligned}
$$

Obviously, $f_{A}$ is full. We have

$$
\begin{aligned}
& \left(R_{f}\right)_{s}\left(h_{1}\right)=\left\{h_{1}, h_{3}, h_{5}\right\}, \\
& \left(R_{f}\right)_{s}\left(h_{2}\right)=\left\{h_{2}, h_{4}, h_{5}\right\}, \\
& \left(R_{f}\right)_{s}\left(h_{3}\right)=\left\{h_{1}, h_{3}, h_{5}\right\}, \\
& \left(R_{f}\right)_{s}\left(h_{4}\right)=\left\{h_{2}, h_{4}\right\}, \\
& \left(R_{f}\right)_{s}\left(h_{5}\right)=\left\{h_{1}, h_{2}, h_{3}, h_{5}\right\} .
\end{aligned}
$$


Let $X=\left\{h_{2}, h_{4}, h_{6}\right\}$. We have

$$
\begin{aligned}
& \underline{\operatorname{apr}}_{P}(X)=\left\{h_{2}, h_{4}, h_{6}\right\}, \\
& \operatorname{apr}_{P}^{\prime}(X)=\left\{h_{4}\right\}, \\
& \operatorname{apr}_{P}^{\prime \prime}(X)=\left\{h_{2}, h_{4}\right\}, \\
& \overline{\operatorname{apr}}_{P}(X)=\left\{h_{2}, h_{4}, h_{5}, h_{6}\right\}, \\
& \overline{\operatorname{apr}}_{P}^{\prime}(X)=\left\{h_{2}, h_{4}, h_{5}\right\}, \\
& \overline{\operatorname{apr}}_{P}^{\prime \prime}(X)=\left\{h_{1}, h_{2}, h_{3}, h_{4}, h_{5}\right\} .
\end{aligned}
$$

Thus,

$$
\begin{aligned}
& \underline{\operatorname{apr}}_{P}(X) \neq \underline{\operatorname{apr}}_{P}^{\prime}(X), \\
& \underline{\operatorname{apr}}_{P}^{\prime}(X) \neq \operatorname{apr}_{P}^{\prime \prime}(X), \\
& \overline{\operatorname{apr}}_{P}(X) \neq \overline{\operatorname{apr}}_{P}^{\prime}(X), \\
& \overline{\operatorname{apr}}_{P}^{\prime}(X) \neq \overline{\operatorname{apr}}_{P}^{\prime \prime}(X) .
\end{aligned}
$$

By Proposition 16 and Lemmas 23, 24, and 25, we have Theorem 27.

Theorem 27. Let $f_{A}$ be a soft set over $U$, and let $P=\left(U, f_{A}\right)$ be a soft approximation space. Then, the following properties hold for any $X \in 2^{U}$.

(1) If $f_{A}$ is full, then

$$
\begin{aligned}
\underline{a p r}_{P}^{\prime \prime \prime}(X) & \subseteq \underline{\operatorname{apr}}_{P}^{\prime}(X) \subseteq \underline{\operatorname{apr}}_{P}^{\prime \prime}(X) \subseteq X \\
& \subseteq \overline{\operatorname{apr}}_{P}^{\prime \prime}(X) \subseteq \overline{\operatorname{apr}}_{P}^{\prime}(X) \subseteq \overline{\operatorname{apr}}_{P}^{\prime \prime \prime}(X)
\end{aligned}
$$

(2) If $f_{A}$ is full and keeping union, then

$$
\begin{aligned}
\underline{a p r}_{P}^{\prime \prime \prime}(X) & \subseteq \underline{\operatorname{apr}}_{P}^{\prime}(X) \subseteq \underline{\operatorname{apr}}{ }_{P}^{\prime \prime}(X) \subseteq \underline{\operatorname{apr}} \underline{P}_{P}(X) \\
& \subseteq X \subseteq \overline{\operatorname{apr}}_{P}^{\prime \prime}(X) \subseteq \overline{\operatorname{apr}}_{P}^{\prime}(X) \\
& \subseteq \overline{\operatorname{apr}}_{P}^{\prime \prime \prime}(X) \subseteq \overline{\operatorname{apr}}_{P}(X) .
\end{aligned}
$$

3.4. The Relationship between Soft Rough Approximation Operators and Pawlak Rough Approximation Operators. In this section, we shall explore the relationship between soft rough approximation operators and Pawlak rough approximation operators.

Definition 28. Let $R$ be an equivalence relation on $U$. Define a mapping $f_{R}: A \rightarrow 2^{U}$ by

$$
f_{R}(a)=[a]_{R}
$$

for each $a \in A$, where $A=U$. Then, $\left(f_{R}\right)_{A}$ is called the soft set induced by $R$ on $U$.
Theorem 29 (see [10]). Let $R$ be an equivalence relation on $U$, let $\left(f_{R}\right)_{A}$ be the soft set induced by $R$ on $U$, and let $P=$ $\left(U,\left(f_{R}\right)_{A}\right)$ be a soft approximation space. Then, for each $X \in$ $2^{U}$,

$$
\underline{R}(X)=\underline{a p r}_{P}(X), \quad \bar{R}(X)=\overline{\operatorname{apr}}_{P}(X) .
$$

Thus, in this case, $X \in 2^{U}$ is a Pawlak rough set if and only if $X$ is a soft $P$-rough set.

By Proposition 16 and Lemmas 23 and 25, we have Theorem 30.

Theorem 30. Let $f_{A}$ be a partition soft set over $U$, and let $P=\left(U, f_{A}\right)$ be a soft approximation space. Then, the following properties hold for any $X \in 2^{U}$.

(1) $\underline{R}_{f}(X)=\underline{\operatorname{apr}}_{P}(X)=\underline{\operatorname{apr}}_{P}^{\prime}(X)=\underline{\operatorname{apr}}_{P}^{\prime \prime}(X)=\underline{\operatorname{apr}_{P}^{\prime \prime \prime}}(X)$;

(2) $\overline{R_{f}}(X)=\overline{\operatorname{apr}}_{P}(X)=\overline{\operatorname{apr}}_{P}^{\prime}(X)=\overline{\operatorname{apr}}_{P}^{\prime \prime}(X)=\overline{\operatorname{apr}}_{P}^{\prime \prime \prime}(X)$,

where $R_{f}(X)$ and $\overline{R_{f}}(X)$ are the Pawlak rough approximations of $X$.

Corollary 31. Let $f_{A}$ be a full soft set over $U$, and let $P=$ $\left(U, f_{A}\right)$ be a soft approximation space. Then,

(1) every soft $P^{\prime \prime \prime}$-definable set is a soft $P^{\prime}$-definable set.

(2) every soft $P^{\prime}$-definable set is a soft $P^{\prime \prime}$-definable set.

Remark 32. Theorems 29 and 30 illustrate that Pawlak's rough set models can be viewed as a special case of soft rough sets.

Remark 33. Example 4.6 in [10] illustrates that a soft rough approximation is a worth considering alternative to the rough approximation. Soft rough sets could provide a better approximation than rough sets do.

\section{The Relationships between Soft Sets and Topologies}

Let $f_{A}$ be a soft set over $U$, and let $P=\left(U, f_{A}\right)$ be a soft approximation space. Denote

$$
\begin{aligned}
& \tau_{f}=\left\{X \in 2^{U}: X=\underline{\operatorname{apr}_{P}}(X)\right\}, \\
& \sigma_{f}=\left\{X \in 2^{U}: X=\overline{\operatorname{apr}}_{P}(X)\right\} ; \\
& \tau_{f}^{\prime}=\left\{X \in 2^{U}: X=\underline{\operatorname{apr}_{P}^{\prime}}(X)\right\}, \\
& \sigma_{f}^{\prime}=\left\{X \in 2^{U}: X=\overline{\operatorname{apr}}_{P}^{\prime}(X)\right\} ; \\
& \tau_{f}^{\prime \prime}=\left\{X \in 2^{U}: X=\underline{\operatorname{apr}}_{P}^{\prime \prime}(X)\right\}, \\
& \sigma_{f}^{\prime \prime}=\left\{X \in 2^{U}: X=\overline{\operatorname{apr}}_{P}^{\prime \prime}(X)\right\} ; \\
& \tau_{f}^{\prime \prime \prime}=\left\{X \in 2^{U}: X=\underline{\operatorname{apr}}_{P}^{\prime \prime \prime}(X)\right\}, \\
& \sigma_{f}^{\prime \prime \prime}=\left\{X \in 2^{U}: X=\overline{\operatorname{apr}}_{P}^{\prime \prime \prime}(X)\right\} .
\end{aligned}
$$


4.1. The First Sort of Topologies Induced by a Soft Set and Related Results. By Propositions 13 and 14, we have Theorem 34.

Theorem 34. Let $f_{A}$ be a full and keeping intersection or a partition soft set over $U$ and let $P=\left(U, f_{A}\right)$ be a soft approximation space. Then $\tau_{f}$ is a topology on $U$.

Remark 35. Let $f_{A}$ be a full and keeping union soft set over $U$, and let $P=\left(U, f_{A}\right)$ be an soft approximation space. Then, by Proposition 14(5), $\sigma_{f}=\{\emptyset, U\}$ is a indiscrete topology on $U$.

The following theorem gives the structure of the first sort of topologies induced by a soft set.

Theorem 36. Let $f_{A}$ be a full and keeping intersection soft set over $U$, let $P=\left(U, f_{A}\right)$ be a soft approximation space, and let $\tau_{f}$ be the topology induced by $f_{A}$ on $U$. Then,

(1)

$$
\left\{\overline{a p r}_{P}(X): X \in 2^{U}\right\} \subseteq \tau_{f}=\left\{\underline{a p r}_{P}(X): X \in 2^{U}\right\}
$$

(2)

$$
\tau_{f} \supseteq\{f(a): a \in A\}
$$

(3) if $f_{A}$ is topological, then

$$
\tau_{f}=\{f(a): a \in A\}
$$

(4) $\underline{a p r}_{P}$ is an interior operator of $\tau_{f}$.

Proof. (1) By Proposition 13, we have

$$
\left\{\overline{\operatorname{apr}}_{P}(X): X \in 2^{U}\right\} \subseteq \tau_{f} .
$$

Obviously,

$$
\tau_{f} \subseteq\left\{\underline{\operatorname{apr}}_{P}(X): X \in 2^{U}\right\}
$$

Let $Y \in\left\{\underline{\operatorname{apr}}_{P}(X): X \in 2^{U}\right\}$. Then, $Y=\underline{\operatorname{apr}}_{P}(X)$ for some $X \in 2^{U}$. By Proposition 13, $\operatorname{apr}_{P}\left(\operatorname{apr}_{P}(X)\right)=\operatorname{apr}_{P}(X)$. This implies $Y \in \tau_{f}$. Thus,

$$
\tau_{f} \supseteq\left\{\underline{\operatorname{apr}}_{P}(X): X \in 2^{U}\right\}
$$

Hence,

$$
\left\{\overline{\operatorname{apr}}_{P}(X): X \in 2^{U}\right\} \subseteq \tau_{f}=\left\{\underline{\operatorname{apr}}_{P}(X): X \in 2^{U}\right\} .
$$

(2) For each $a \in A$, by Proposition 13,

$$
\begin{aligned}
& \underset{\operatorname{apr}_{P}}{\operatorname{ar}_{P}}(f(a)) \\
& \quad=\bigcup\left\{f\left(a^{\prime}\right): a^{\prime} \in A, f\left(a^{\prime}\right) \subseteq f(a)\right\} \subseteq f(a) .
\end{aligned}
$$

Then, $f(a)=\underline{\operatorname{apr}}_{P}(f(a))$. So $f(a) \in \tau_{f}$. Thus,

$$
\{f(a): a \in A\} \subseteq \tau_{f} .
$$

(3) Suppose that $X \in \tau_{f}$. If $X=\emptyset$, by $f_{A}$ is topological, there exists $a \in A$ such that $X=f(a)$. If $X \neq \emptyset$, for each $x \in X$, $X=\underline{\operatorname{apr}}_{P}(X)$, there exists $a_{x} \in A$ such that $x \in f\left(a_{x}\right) \subseteq X$. Then,

$$
X=\bigcup_{x \in X}\{x\} \subseteq \bigcup_{x \in X} f\left(a_{x}\right) \subseteq X .
$$

So, $X=\bigcup_{x \in X} f\left(a_{x}\right)$. Since $f_{A}$ is keeping union, then

$$
\bigcup_{x \in X} f\left(a_{x}\right)=f(a) \quad \text { for some } a \in A
$$
A\}.

This implies $X \in\{f(a): a \in A\}$. Thus, $\tau_{f} \subseteq\{f(a): a \in$ By (1), $\tau_{f} \supseteq\{f(a): a \in A\}$.

Hence,

$$
\tau_{f}=\{f(a): a \in A\} .
$$

(4) It suffices to show that

$$
\underline{\operatorname{apr}}_{P}(X)=\operatorname{int}(X) \text { for each } X \in 2^{U} \text {. }
$$

By (1), $\underline{\operatorname{apr}}_{P}(X) \in \tau_{f}$. By Proposition 13, $\underline{\operatorname{apr}}_{P}(X) \subseteq X$. Thus

$$
\underset{P}{\operatorname{apr}}(X) \subseteq \operatorname{int}(X) .
$$

Conversely, for each $Y \in \tau_{f}$ with $Y \subseteq X$, we have $Y=$ $\underline{\operatorname{apr}}_{P}(Y) \subseteq \underline{\operatorname{apr}}_{P}(X)$ by Proposition 13. Thus,

$$
\operatorname{int}(X)=\cup\left\{Y: Y \in \tau_{f}, Y \subseteq X\right\} \subseteq \underline{\operatorname{apr}}_{P}(X) .
$$

Hence,

$$
\underset{P}{\operatorname{apr}}(X)=\operatorname{int}(X) \text {. }
$$

Definition 37. Let $\tau$ be a topology on $U$. Put $\tau=\left\{U_{a}: a \in A\right\}$, where $A$ is the set of indexes. Define a mapping $f_{\tau}: A \rightarrow 2^{U}$ by $f_{\tau}(a)=U_{a}$ for each $a \in A$. Then, the soft set $\left(f_{\tau}\right)_{A}$ over $U$ is called the soft set induced by $\tau$ on $U$.

Definition 38. Let $(U, \mu)$ be a topological space. If there exists a full and keeping intersection or a partition soft set $f_{A}$ over $U$ such that $\tau_{f}=\mu$, then $(U, \mu)$ is called a soft approximating space.

The following proposition can easily be proved.

Proposition 39. (1) Let $\tau$ be a topology on $U$, and let $\left(f_{\tau}\right)_{A}$ be the soft set induced by $\tau$ on $U$. Then, $\left(f_{\tau}\right)_{A}$ is a full, keeping intersection, and keeping union soft set over $U$.

(2) Let $\tau_{1}$ and $\tau_{2}$ be two topologies on $U$, and let $\left(f_{\tau_{1}}\right)_{A_{1}}$ and $\left(f_{\tau_{2}}\right)_{A_{2}}$ be two soft sets induced, respectively, by $\tau_{1}$ and $\tau_{2}$ on $U$. If $\tau_{1} \subseteq \tau_{2}$, then

$$
\left(f_{\tau_{1}}\right)_{A_{1}} \tilde{\mathcal{C}}\left(f_{\tau_{2}}\right)_{A_{2}} .
$$


Theorem 40. Let $\tau$ be a topology on $U$, let $\left(f_{\tau}\right)_{A}$ be the soft set induced by $\tau$ on $U$, and let $\tau_{f_{\tau}}$ be the topology induced by $\left(f_{\tau}\right)_{A}$ on $U$. Then, $\tau=\tau_{f_{\tau}}$.

Proof. Put $\tau=\left\{U_{a}: a \in A\right\}$; then, $f_{\tau}: A \rightarrow 2^{U}$ is a mapping, where $f_{\tau}(a)=U_{a}$ for each $a \in A$. By Proposition $39,\left(f_{\tau}\right)_{A}$ is full, keeping intersection, and keeping union.

By Theorem 36, $\tau_{f_{\tau}}=\left\{f_{\tau}(a): a \in A\right\}$.

Hence, $\tau_{f_{\tau}}=\tau$.

Corollary 41. Every topological space on the initial universe is a soft approximating space.

Theorem 42. Let $(U, \tau)$ be a topological space. Then, there exists a full, keeping intersection, and keeping union soft set $f_{A}$ over $U$ such that

$$
\underline{\operatorname{apr}}_{P}(X)=\operatorname{int}(X) \quad \text { for each } X \in 2^{U},
$$

where $P=\left(U, f_{A}\right)$ is a soft approximation space.

Proof. Put $\tau=\left\{U_{a}: a \in A\right\}$, where $A$ is the set of indexes. Define a mapping $f: A \rightarrow 2^{U}$ by

$$
f(a)=U_{a} \quad \text { for each } a \in A .
$$

By Proposition 39, $f_{A}$ is full, keeping intersection, and keeping union.

Let $X \in 2^{U}$. For each $x \in \operatorname{apr}_{p}(X), x \in f(a) \subseteq X$ for some $a \in A$. Then, $x \in U_{a} \subseteq \bar{X}$ with $U_{a} \in \tau$. This implies $x \in \operatorname{int}(X)$.

Conversely, for each $x \in \operatorname{int}(X)$, there exists an open neighborhood $W$ of $x$ in $U$ such that $W \subseteq X$. So, $W=U_{a}$ for some $a \in A$. This implies $x \in f(a) \subseteq X$. Thus, $x \in \operatorname{apr}_{p}(X)$.

Hence, $\operatorname{apr}_{P}(X)=\operatorname{int}(X)$.

Theorem 43. Let $f_{A}$ be a full and keeping intersection soft set over $U$, let $\tau_{f}$ be the topology induced by $f_{A}$ on $U$, and let $\left(f_{\tau_{f}}\right)_{B}$ be the soft set induced by $\tau_{f}$ on $U$. Then,

(1)

$$
f_{A} \widetilde{c}\left(f_{\tau_{f}}\right)_{B} .
$$

(2) If $f_{A}$ is topological, then

$$
f_{A}=\left(f_{\tau_{f}}\right)_{B} .
$$

Proof. (1) By Theorem 36, $\tau_{f} \supseteq\{f(a): a \in A\}$. Denote

$$
\begin{gathered}
\tau_{f}=\left\{U_{a}: a \in B\right\}, \quad \text { where } A \subseteq B, \\
U_{a}=f(a) \quad \text { for each } a \in A .
\end{gathered}
$$

Thus $f_{\tau_{f}}$ is a mapping given by $f_{\tau_{f}}: B \rightarrow 2^{U}$, where $f_{\tau_{f}}(a)=$ $U_{a}$ for each $a \in B$.

Hence, $f_{A} \tilde{C}\left(f_{\tau_{f}}\right)_{B}$.

(2) Since $f_{A}$ is topological, then by Theorem $36, A=B$. Hence,

$$
f_{A}=\left(f_{\tau_{f}}\right)_{B} .
$$

4.2. The Second Sort of Topologies Induced by a Soft Set. By Proposition 19, we have Theorem 44.

Theorem 44. Let $f_{A}$ be a full soft set over $U$, and let $P=$ $\left(X, f_{A}\right)$ be a soft approximation space. Then, $\tau_{f}^{\prime}$ is a topology on $U$.

Definition 45. Let $\tau$ be a topology on $U$.

(1) $\tau$ is called an Alexandrov topology on $U$, if $\tau$ is closed for arbitrary intersections.

(2) $(U, \tau)$ is called an Alexandrov space, if $\tau$ is an Alexandrov topology on $U$.

(3) $\tau$ is called a pseudodiscrete topology on $U$, if $A \in \tau$ if and only if $U-A \in \tau$.

Obviously, every pseudodiscrete topology is an Alexandrov topology.

The following theorem gives the structure of the second sort of topologies induced by a soft set.

Theorem 46. Let $f_{A}$ be a full soft set over $U$, and let $\tau_{f}^{\prime}$ be the topology induced by $f_{A}$ on $U$. Then, $\left(U, \tau_{f}^{\prime}\right)$ is an Alexandrov space.

Proof. By Proposition 16, $R_{f}$ is reflexive and symmetric. Then, by Proposition 5 in $[16], \tau_{f}^{\prime}$ is a pseudodiscrete topology on $U$.

Thus,

$$
\left(U, \tau_{f}^{\prime}\right) \text { is an Alexandrov space. }
$$

\subsection{The Third Sort of Topologies Induced by a Soft Set}

Example 47. Let $f_{A}$ be a full soft set over $U$ and let $P=\left(U, f_{A}\right)$ be a soft approximation space in Example 26.

Let $Y=\left\{h_{2}, h_{4}\right\}$, and $Z=\left\{h_{1}, h_{2}, h_{3}, h_{5}\right\}$. We have

$$
\begin{aligned}
& \underline{\operatorname{apr}}_{P}^{\prime \prime}(Y)=Y, \\
& \underline{\operatorname{apr}}_{P}^{\prime \prime}(Z)=Z, \\
& \underline{\operatorname{apr}}_{P}^{\prime \prime}(Y \cap Z)=\emptyset \neq Y \cap Z .
\end{aligned}
$$

Thus, $\tau_{f}^{\prime \prime}$ is not a topology on $U$.

By Proposition 21, we have Theorem 48.

Theorem 48. Let $f_{A}$ be a full soft set over $U$, and let $P=$ $\left(U, f_{A}\right)$ be a soft approximation space. Then, $\tau_{f}^{\prime \prime \prime}$ is a topology on $U$.

4.4. The Relationships among Three Sorts of Topologies Induced by a Soft Set and Related Results. By Theorem 27, we have Theorem 49, which illustrates relationships among three sorts of topologies induced by a soft set. 
Theorem 49. (1) If $f_{A}$ is a full soft set over $U$, then

$$
\tau_{f}^{\prime \prime \prime} \subseteq \tau_{f}^{\prime}
$$

(2) If $f_{A}$ is a full and keeping intersection soft set over $U$, then

$$
\tau_{f}^{\prime \prime \prime} \subseteq \tau_{f}^{\prime} \subseteq \tau_{f}
$$

(3) If $f_{A}$ is a partition soft set over $U$, then

$$
\tau_{f}=\tau_{f}^{\prime}=\tau_{f}^{\prime \prime \prime}
$$

By Theorem 36, Proposition 39 and Theorem 49, we have Theorem 50.

Theorem 50. Let $\tau$ be a topology on $U$, let $\left(f_{\tau}\right)_{A}$ be the soft set induced by $\tau$ on $U$, and let $\tau_{f_{\tau}}, \tau_{f_{\tau}}^{\prime}$ and $\tau_{f_{\tau}}^{\prime \prime \prime}$ be the topology induced, respectively, by $\left(f_{\tau}\right)_{A}$ on $U$. Then

$$
\tau=\tau_{f_{\tau}} \supseteq \tau_{f_{\tau}}^{\prime} \supseteq \tau_{f_{\tau}}^{\prime \prime \prime}
$$

By Proposition 39 and Theorems 43 and 49, we have Theorem 51.

Theorem 51. (1) If $f_{A}$ is a full soft set over $U$, let $\tau_{f}^{\prime}$ and $\tau_{f}^{\prime \prime \prime}$ be the topologies induced, respectively, by $f_{A}$ on $U$, and let $\left(f_{\tau_{f}^{\prime}}\right)_{C}\left(\right.$ resp., $\left.\left(f_{\tau_{f}^{\prime \prime \prime}}\right)_{D}\right)$ be the soft set induced by $\tau_{f}^{\prime}$ (resp., $\left.\tau_{f}^{\prime \prime \prime}\right)$ on $U$. Then,

$$
\left(f_{\tau_{f}^{\prime}}\right)_{C} \tilde{}\left(f_{\tau_{f}^{\prime \prime \prime}}\right)_{D}
$$

(2) Let $f_{A}$ be a full and keeping intersection soft set over $U$, let $\tau_{f}, \tau_{f}^{\prime}$, and $\tau_{f}^{\prime \prime \prime}$ be the topologies induced respectively by $f_{A}$ on $U$ and let $\left(f_{\tau_{f}}\right)_{B}$ (resp., $\left.\left(f_{\tau_{f}^{\prime}}\right)_{C},\left(f_{\tau_{f}^{\prime \prime \prime}}\right)_{D}\right)$ be the soft set induced by $\tau_{f}$ (resp., $\left.\tau_{f}^{\prime}, \tau_{f}^{\prime \prime \prime}\right)$ on $U$. Then,

(a)

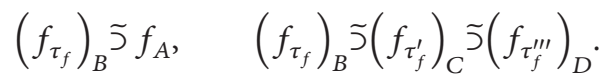

(b) If $f_{A}$ is keeping union, then

$$
f_{A}=\left(f_{\tau_{f}}\right)_{B} \widetilde{\supset}\left(f_{\tau_{f}^{\prime}}\right)_{C} \widetilde{\supset}\left(f_{\tau_{f}^{\prime \prime \prime}}\right)_{D}
$$

(3) Let $f_{A}$ be a partition soft set over $U$, let $\tau_{f_{\tau}}, \tau_{f_{\tau}}^{\prime}$ and $\tau_{f_{\tau}}^{\prime \prime \prime}$ be the topologies induced, respectively, by $f_{A}$ on $U$ and let $\left(f_{\tau_{f}}\right)_{B}$ (resp., $\left.\left(f_{\tau_{f}^{\prime}}\right)_{C},\left(f_{\tau_{f}^{\prime \prime \prime}}\right)_{D}\right)$ be the soft set induced by $\tau_{f}\left(\right.$ resp., $\left.\tau_{f}^{\prime}, \tau_{f}^{\prime \prime \prime}\right)$ on $U$. Then,

$$
\left(f_{\tau_{f}}\right)_{B} \tilde{\supset} f_{A}, \quad\left(f_{\tau_{f}}\right)_{B}=\left(f_{\tau_{f}^{\prime}}\right)_{C}=\left(f_{\tau_{f}^{\prime \prime \prime}}\right)_{D}
$$

Example 52. Let $f_{A}$ be a partition soft set over $U$ in Example 11, let $\tau_{f}$ be the topology induced by $f_{A}$ on $U$, and let $\left(f_{\tau_{f}}\right)_{B}$ be the soft set induced by $\tau_{f}$ on $U$. We have

$$
\begin{aligned}
& \tau_{f}=\left\{U_{a}: a \in B\right\}, \\
& \text { where } B=\left\{a_{1}, a_{2}, \ldots, a_{16}\right\}, \\
& U_{a_{1}}=f\left(a_{1}\right)=\left\{h_{1}, h_{2}\right\}, \\
& U_{a_{2}}=f\left(a_{2}\right)=\left\{h_{5}\right\}, \\
& U_{a_{3}}=f\left(a_{3}\right)=\left\{h_{3}\right\}, \\
& U_{a_{4}}=f\left(a_{4}\right)=\left\{h_{4}\right\}, \\
& U_{a_{5}}=\left\{h_{3}, h_{4}\right\}, \\
& U_{a_{6}}=\left\{h_{3}, h_{5}\right\}, \\
& U_{a_{7}}=\left\{h_{4}, h_{5}\right\}, \\
& U_{a_{8}}=\left\{h_{1}, h_{2}, h_{3}\right\}, \\
& U_{a_{9}}=\left\{h_{1}, h_{2}, h_{4}\right\}, \\
& U_{a_{10}}=\left\{h_{1}, h_{2}, h_{5}\right\}, \\
& U_{a_{11}}=\left\{h_{3}, h_{4}, h_{5}\right\}, \\
& U_{a_{12}}=\left\{h_{1}, h_{2}, h_{3}, h_{4}\right\}, \\
& U_{a_{13}}=\left\{h_{1}, h_{2}, h_{3}, h_{5}\right\}, \\
& U_{a_{14}}=\left\{h_{1}, h_{2}, h_{4}, h_{5}\right\}, \\
& U_{a_{15}}=\emptyset, \\
& U_{a_{16}}=U .
\end{aligned}
$$

Obviously,

$$
f_{A} \widetilde{\subset}\left(f_{\tau_{f}}\right)_{B}, \quad f_{A} \widetilde{\nsupseteq}\left(f_{\tau_{f}}\right)_{B} .
$$

Thus,

$$
f_{A} \neq\left(f_{\tau_{f}}\right)_{B}
$$

\section{The Related Properties of Soft Rough Sets}

In this section, four sorts of soft rough sets based on four pairs of soft rough approximations are investigated.

For $\mathscr{A}, \mathscr{B} \subseteq 2^{U}$, we denote

$$
\mathscr{A} \backslash \mathscr{B}=\left\{X \in 2^{U}: X \in \mathscr{A}, X \notin \mathscr{B}\right\} .
$$

Lemma 53 (see [10]). Let $f_{A}$ be a soft set over $U$ and let $P=$ $\left(U, f_{A}\right)$ be a soft approximation space. Then for each $X \in 2^{U}$,

$$
X \text { is a soft } P \text {-rough set } \Longleftrightarrow \overline{a p r}_{P}(X) \subseteq X \text {. }
$$

By Corollary 31, we have the following Lemma 54. 
Lemma 54. Let $f_{A}$ be a full soft set over $U$, and let $P=\left(U, f_{A}\right)$ be a soft approximation space. Then,

(1) every soft $P^{\prime}$-rough set is a soft $P^{\prime \prime \prime}$-rough set.

(2) every soft $P^{\prime \prime}$-rough set is a soft $P^{\prime}$-rough set.

By Theorem 27, we have Lemma 55.

Lemma 55. Let $f_{A}$ be a full and keeping union soft set over $U$, and let $P=\left(U, f_{A}\right)$ be a soft approximation space. If $X \in 2^{U}$ is a soft $P$-definable set, then,

$$
X \in \sigma_{f}^{\prime \prime \prime} \subseteq \sigma_{f}^{\prime} \subseteq \sigma_{f}^{\prime \prime} .
$$

The following theorem gives the structure of soft rough sets.

Theorem 56. Let $f_{A}$ be a soft set over $U$, and let $P=\left(U, f_{A}\right)$ be a soft approximation space. Denote

$$
\begin{aligned}
& \Omega=\left\{X \in 2^{U}: X \text { is a soft } P \text {-rough set }\right\}, \\
& \Omega^{\prime}=\left\{X \in 2^{U}: X \text { is a soft } P^{\prime} \text {-rough set }\right\}, \\
& \Omega^{\prime \prime}=\left\{X \in 2^{U}: X \text { is a soft } P^{\prime \prime}{ }_{\text {-rough set }\},}\right. \\
& \Omega^{\prime \prime \prime}=\left\{X \in 2^{U}: X \text { is a soft } P^{\prime \prime \prime}{ }_{\text {-rough } \text { set }}\right\} .
\end{aligned}
$$

(1) If $f_{A}$ is full, then

(a)

$$
\sigma_{f}=2^{U} \backslash \Omega ;
$$

(b)

$$
\Omega^{\prime \prime} \subseteq \Omega^{\prime} \subseteq \Omega^{\prime \prime \prime}
$$

(c)

$$
2^{U} \backslash \tau_{f}^{\prime} \subseteq \Omega^{\prime}, \quad 2^{U} \backslash \tau_{f}^{\prime \prime} \subseteq \Omega^{\prime \prime}, \quad 2^{U} \backslash \tau_{f}^{\prime \prime \prime} \subseteq \Omega^{\prime \prime \prime} ;
$$

(d)

$$
\begin{aligned}
& \tau_{f}^{\prime} \cap \sigma_{f}^{\prime}=2^{U} \backslash \Omega^{\prime}, \\
& \tau_{f}^{\prime \prime} \cap \sigma_{f}^{\prime \prime}=2^{U} \backslash \Omega^{\prime \prime}, \\
& \tau_{f}^{\prime \prime \prime} \cap \sigma_{f}^{\prime \prime \prime}=2^{U} \backslash \Omega^{\prime \prime \prime} .
\end{aligned}
$$

(2) If $f_{A}$ is full and keeping union, then
(a) $2^{U} \backslash \sigma_{f}^{\prime \prime} \subseteq 2^{U} \backslash \sigma_{f}^{\prime} \subseteq 2^{U} \backslash \sigma_{f}^{\prime \prime \prime} \subseteq 2^{U} \backslash \sigma_{f} \subseteq \Omega$;
(b) $\sigma_{f}=\{\emptyset, U\}=2^{U} \backslash \Omega$.

(3) If $f_{A}$ is full and keeping intersection, then

(a) $\sigma_{f}=2^{U} \backslash \Omega \subseteq \tau_{f}$.
Proof. These hold by Proposition 14 and Lemmas 53, 54, and 55.

Theorem 57. Let $f_{A}$ be a soft set over $U$ and let $P=\left(U, f_{A}\right)$ be a soft approximation space. Then, for $X \in 2^{U}$, one has

$$
\begin{aligned}
& \operatorname{Pos}_{P}^{\prime}(U-X)=\operatorname{Neg}_{P}^{\prime}(X), \\
& \operatorname{Pos}_{P}^{\prime \prime}(U-X)=\operatorname{Neg}_{P}^{\prime \prime}(X), \\
& \operatorname{Pos}_{P}^{\prime \prime \prime}(U-X)=\operatorname{Neg}_{P}^{\prime \prime \prime}(X) .
\end{aligned}
$$

Proof. This is obtained from Propositions 19, 20, and 21.

By Proposition 14 and Theorem 27, we have Theorem 58.

Theorem 58. Let $f_{A}$ be a soft set over $U$, and let $P=\left(U, f_{A}\right)$ be a soft approximation space. Then, for $X \in 2^{U}$, one has the following.

(1) If $f_{A}$ be a full, then

$$
\operatorname{Bnd}_{P}^{\prime \prime}(X) \subseteq B n d_{P}^{\prime}(X) \subseteq B n d_{P}^{\prime \prime \prime}(X) .
$$

(2) If $f_{A}$ is full and keeping union, then

(a) $\overline{\operatorname{apr}}_{P}^{\prime \prime}(X)-X \subseteq \overline{\operatorname{apr}}_{P}^{\prime}(X)-X \subseteq \overline{\operatorname{apr}}_{P}^{\prime \prime \prime}(X)-X \subseteq$ $\operatorname{Bnd}_{P}(X)$;

(b) $\operatorname{Neg}_{P}(X)=\emptyset$ and $B n d_{P}(X)=U-\operatorname{Pos}_{P}(X)$ where $X \neq \emptyset$.

Remark 59. Theorem 58 illustrates that soft $P^{\prime \prime}$-rough sets could provide a better approximation than soft $P^{\prime}$-rough sets do and soft $P^{\prime}$-rough sets could provide a better approximation than soft $P^{\prime \prime \prime}$-rough sets do.

\section{A Correspondence Relationship}

In this section, we give a one-to-one correspondence relationship in order to reveal the broad application prospect of soft sets.

Definition 60 (see [17]). Let $U$ be a finite set of objects, let $A$ be a finite set of attributes, and let $I$ be a binary relation on from $U$ to $A$. The triple $(U, A, I)$ is called a formal context.

Let $(U, A, I)$ be a formal context. For $u \in U$ and $a \in A$, $u R a$, which is also written as $(u, a) \in R$, means that the object $u$ possesses the attribute $a$.

Denote

$$
\{a\}^{*}=\{u \in U: u I a\}, \quad\{u\}^{*}=\{a \in A: u I a\} .
$$

Definition 61. Let $\mathrm{FC}=(U, A, I)$ be a formal context. Define a mapping $f_{\mathrm{FC}}: A \rightarrow 2^{U}$ by

$$
f_{\mathrm{FC}}(a)=\{a\}^{*}
$$

for each $a \in A$. Then, $\left(f_{\mathrm{FC}}\right)_{A}=\left(f_{\mathrm{FC}}, A\right)$ is called the soft set over $U$ induced by FC. We denote $\left(f_{\mathrm{FC}}\right)_{A}$ by $S_{\mathrm{FC}}$. 
Definition 62. Let $S=f_{A}$ be a soft set over $U$. Define a binary relation $I_{S} \in 2^{U} \times A$ by

$$
(u, a) \in I_{S} \Longleftrightarrow u \in f(a)
$$

for each $u \in U$ and each $a \in A$. Then, $\left(U, A, I_{S}\right)$ is called the formal context induced by $S$. We denote $\left(U, A, I_{S}\right)$ by $\mathrm{FC}_{S}$.

Lemma 63. Let $F C=(U, A, I)$ be a formal context, let $S_{F C}$ be the soft set induced by $F C$, and let $F C_{S_{F C}}$ be the formal context induced by $S_{F C}$. Then,

$$
F C=F C_{S_{F C}} .
$$

Proof. Obviously, $\mathrm{FC}_{S_{\mathrm{FC}}}=\left(U, A, I_{S_{\mathrm{FC}}}\right)$.

For each $(u, a) \in U \times A$,

$$
(u, a) \in I_{S_{\mathrm{FC}}} \Longleftrightarrow u \in f_{\mathrm{FC}}(a) .
$$

For each $a \in A$,

$$
f_{\mathrm{FC}}(a)=\{a\}^{*}=\{u \in U: u I a\}=\{u \in U:(u, a) \in I\} .
$$

Then,

$$
u \in f_{\mathrm{FC}}(a) \Longleftrightarrow(u, a) \in I .
$$

Thus, for each $(u, a) \in U \times A,(u, a) \in I_{S_{\mathrm{FC}}} \Leftrightarrow(u, a) \in I$. This implies $I_{S_{\mathrm{FC}}}=I$.

Hence,

$$
\mathrm{FC}=\mathrm{FC}_{S_{\mathrm{FC}}}
$$

Lemma 64. Let $S=f_{A}$ be a soft set over $U$, let $F C_{S}$ be the formal context induced by $S$, and let

$$
S_{F C_{S}}
$$

be the soft set induced by $F C_{S}$. Then,

$$
S=S_{F C_{S}} \text {. }
$$

Proof. Obviously, $S_{\mathrm{FC}_{S}}=\left(f_{\mathrm{FC}_{S}}, A\right)$.

Since $\mathrm{FC}_{S}=\left(U, A, I_{S}\right)$, then

$f_{\mathrm{FC}_{S}}(a)=\left\{u \in U:(u, a) \in I_{S}\right\} \quad$ for each $a \in A$.

So, $(u, a) \in I_{S} \Leftrightarrow u \in f(a)$.

Obviously, $f(a)=\{u \in U: u \in f(a)\}$ for each $a \in A$.

Thus, for each $a \in A, f_{\mathrm{FC}}(a)=f(a)$.

Hence,

$$
S=S_{\mathrm{FC}_{S}}
$$

Theorem 65. Let

$$
\begin{gathered}
\Gamma=\left\{S=f_{A}: S \text { is a soft set over } U\right\}, \\
\Sigma=\{F C=(U, A, I): F C \text { is a formal context }\} .
\end{gathered}
$$
and $\Sigma$.
Proof. Two mappings $k: \Sigma \rightarrow \Gamma$ and $l: \Gamma \rightarrow \Sigma$ are defined as follows:

$$
\begin{gathered}
k(\mathrm{FC})=S_{\mathrm{FC}}, \quad \text { for any } \mathrm{FC}=(U, A, I) \in \Sigma, \\
l(S)=\mathrm{FC}_{S}, \quad \text { for any } S=f_{A} \in \Gamma .
\end{gathered}
$$

By Lemma 63,

$$
l \circ k=i_{\Sigma}
$$

where $l \circ k$ is the composition of $k$ and $G$, and $i_{\Sigma}$ is the identity mapping on $\Sigma$.

By Lemma 64,

$$
k \circ l=i_{\Gamma}
$$

where $l \circ F$ is the composition of $l$ and $k$, and $i_{\Gamma}$ is the identity mapping on $\Gamma$.

Hence, $F$ and $G$ are both a one-to-one correspondence. This prove that there exists a one-to-one correspondence between $\Sigma$ and $\Gamma$.

Remark 66. Theorem 65 illustrates that we can do formal concept analysis for soft sets or do soft analysis for formal contexts.

\section{Conclusions}

In this paper, we investigated soft rough approximation operators and the problems of combing soft sets with soft rough sets and topologies. Four pairs of soft rough approximation operators were considered, and their properties were given. Four sorts of soft rough sets are defined by using four pairs of soft rough approximation operators, and Pawlak's rough set models can be viewed as a special case of soft rough sets. We researched relationships among soft sets, soft rough sets and topologies, obtained the structure of soft rough sets, and revealed that every topological space on the initial universe is a soft approximating space. We may mention that soft rough sets can be used in object evaluation and group decision making. It should be noted that the use of soft rough sets could, to some extent, automatically reduce the noise factor caused by the subjective nature of the expert's evaluation. We will investigate these problems in future papers.

\section{Acknowledgment}

This work is supported by the National Natural Science Foundation of China (no. 11061004, 11226085), the Science Research Project of Guangxi University for Nationalities (no. 2011QD015) and the Science Foundation of Guangxi College of Finance and Economics (no. 2012ZD001).

\section{References}

[1] Z. Pawlak, Rough Sets: Theoretical Aspects of Reasoning about Data, Kluwer Academic, Boston, Mass, USA, 1991.

[2] D. Molodtsov, "Soft set theory-first results," Computers \& Mathematics with Applications, vol. 37, no. 4-5, pp. 19-31, 1999. 
[3] P. K. Maji, R. Biswas, and A. R. Roy, "Soft set theory," Computers \& Mathematics with Applications, vol. 45, no. 4-5, pp. 555-562, 2003.

[4] P. K. Maji, A. R. Roy, and R. Biswas, "An application of soft sets in a decision making problem," Computers \& Mathematics with Applications, vol. 44, no. 8-9, pp. 1077-1083, 2002.

[5] P. K. Maji, R. Biswas, and A. R. Roy, "Fuzzy soft sets," Journal of Fuzzy Mathematics, vol. 9, no. 3, pp. 589-602, 2001.

[6] A. R. Roy and P. K. Maji, "A fuzzy soft set theoretic approach to decision making problems," Journal of Computational and Applied Mathematics, vol. 203, no. 2, pp. 412-418, 2007.

[7] Y. Jiang, Y. Tang, Q. Chen, J. Wang, and S. Tang, "Extending soft sets with description logics," Computers \& Mathematics with Applications, vol. 59, no. 6, pp. 2087-2096, 2010.

[8] H. Aktaş and N. Çağman, "Soft sets and soft groups," Information Sciences, vol. 177, no. 13, pp. 2726-2735, 2007.

[9] F. Feng, C. Li, B. Davvaz, and M. I. Ali, "Soft sets combined with fuzzy sets and rough sets: a tentative approach," Soft Computing, vol. 14, no. 9, pp. 899-911, 2010.

[10] F. Feng, X. Liu, V. Leoreanu-Fotea, and Y. B. Jun, "Soft sets and soft rough sets," Information Sciences, vol. 181, no. 6, pp. 11251137, 2011.

[11] M. Shabir and M. Naz, "On soft topological spaces," Computers \& Mathematics with Applications, vol. 61, no. 7, pp. 1786-1799, 2011.

[12] X. Ge, Z. Li, and Y. Ge, "Topological spaces and soft sets," Journal of Computational Analysis and Applications, vol. 13, no. 5, pp. 881-885, 2011.

[13] Z. Pawlak and A. Skowron, "Rough sets: some extensions," Information Sciences, vol. 177, no. 1, pp. 28-40, 2007.

[14] Y. Y. Yao and T. Y. Lin, "Generalization of rough sets using modal logic," Intelligent Automation and Soft Computing, vol. 2, no. 2, pp. 103-120, 1996.

[15] W. Zhang, W. Wu, J. Liang, and D. Li, Rough Sets Theorey and Methods, Chinese Scientific, Beijing, China, 2001.

[16] M. Kondo, "On the structure of generalized rough sets," Information Sciences, vol. 176, no. 5, pp. 589-600, 2006.

[17] B. Ganter and R. Wille, Formal Concept Analysis: Mathematical Foundations, Springer, New York, NY, USA, 1999. 


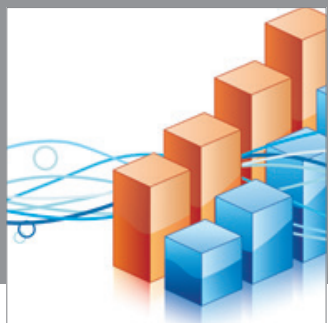

Advances in

Operations Research

mansans

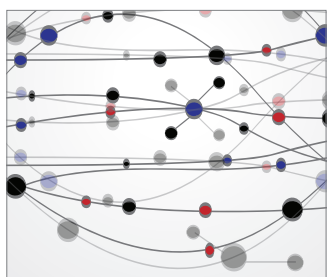

The Scientific World Journal
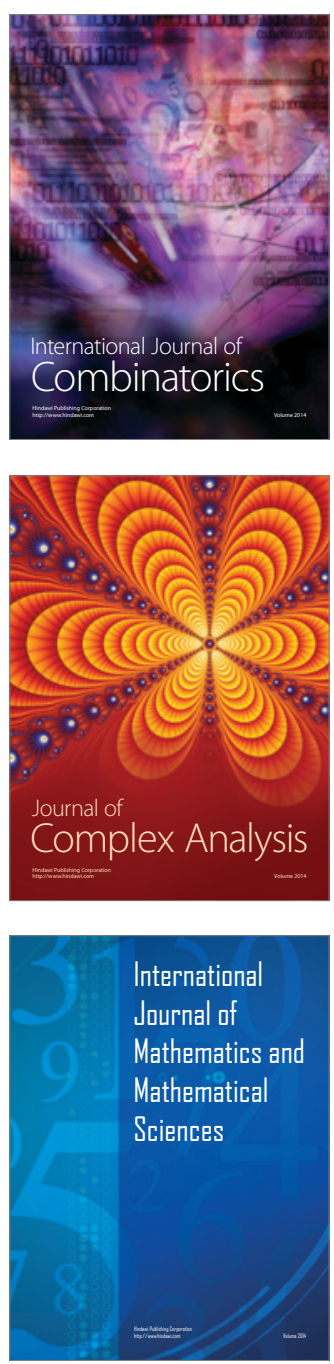
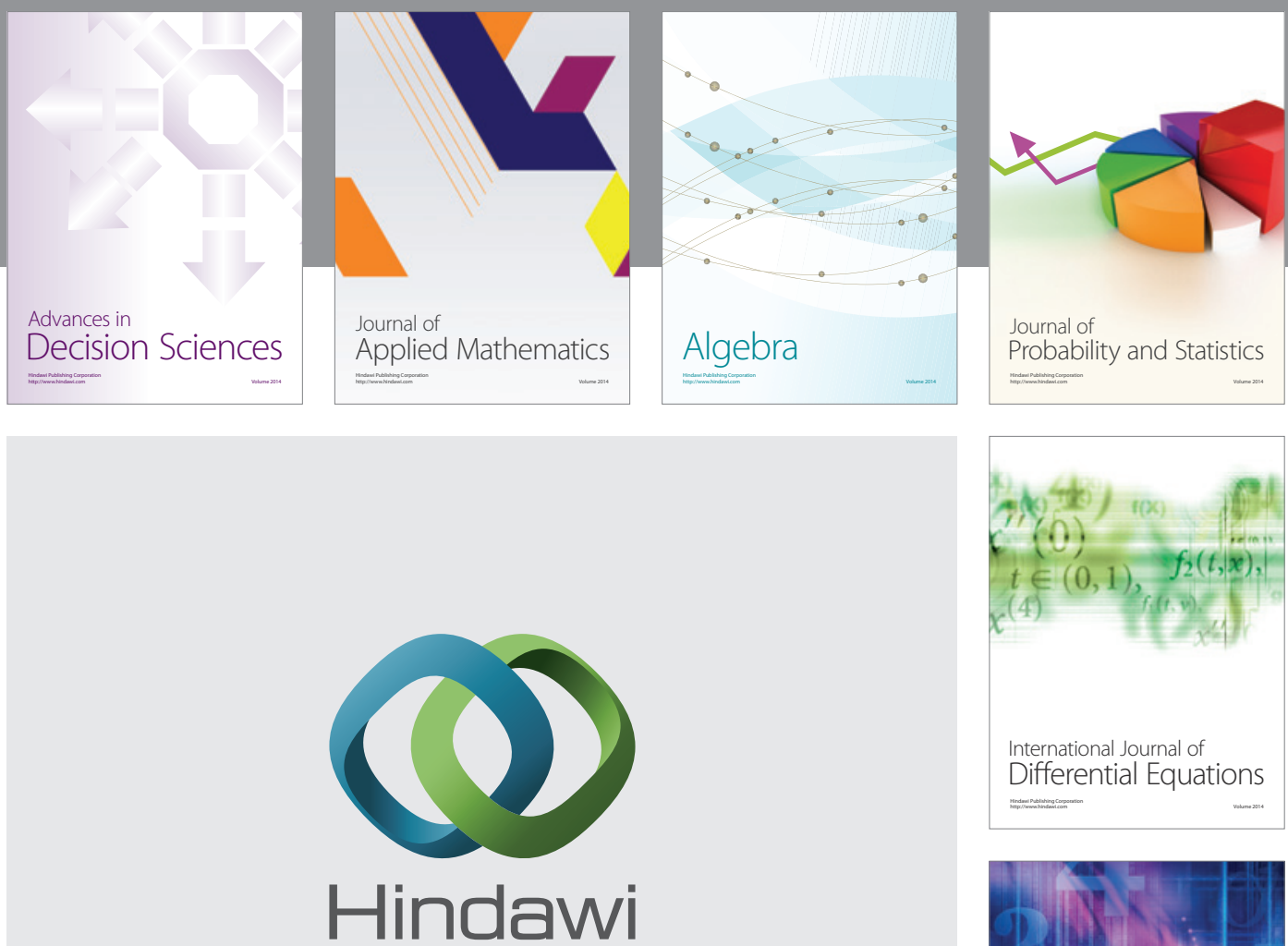

Submit your manuscripts at http://www.hindawi.com
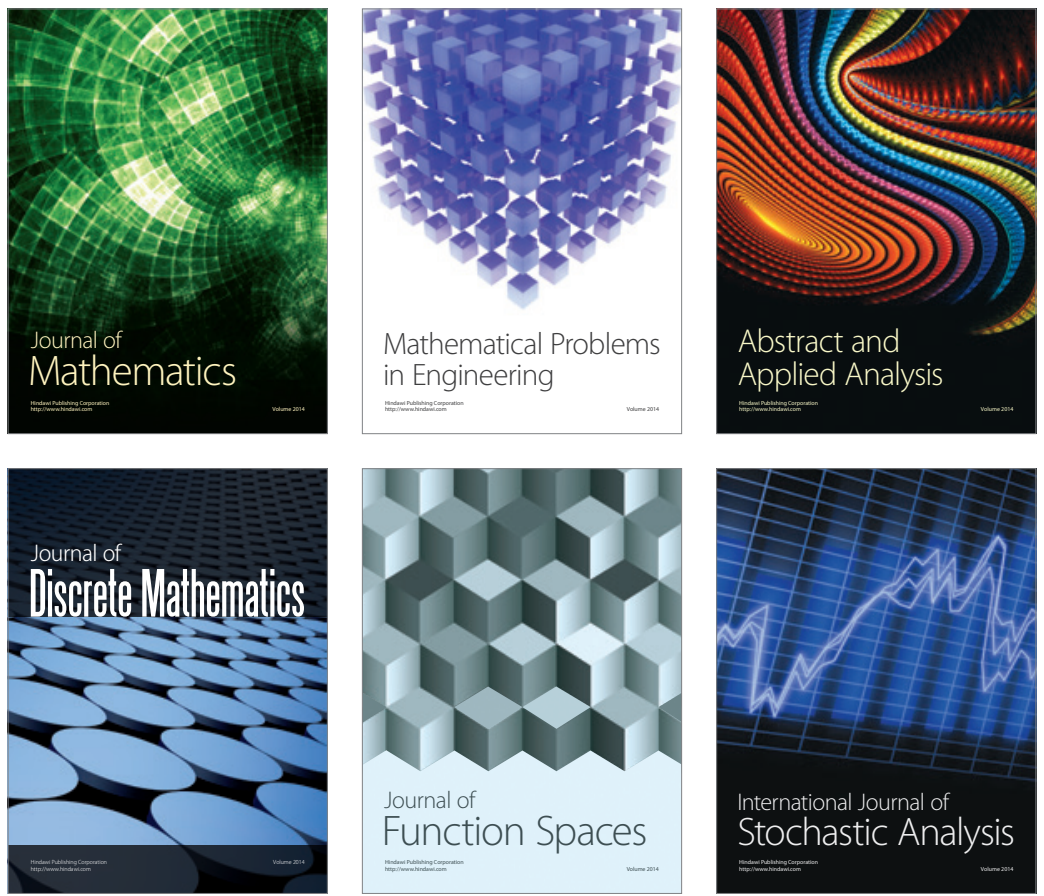

Journal of

Function Spaces

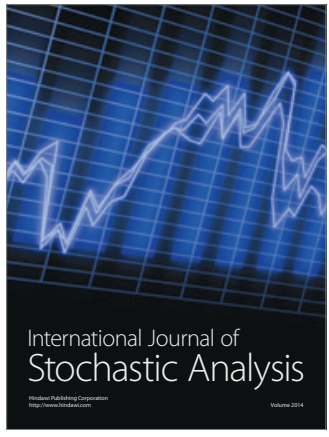

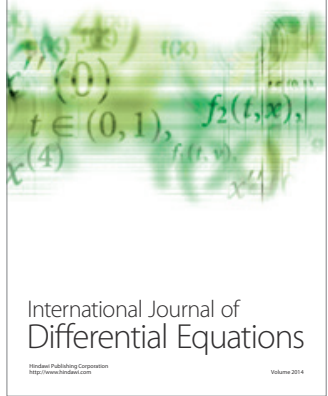
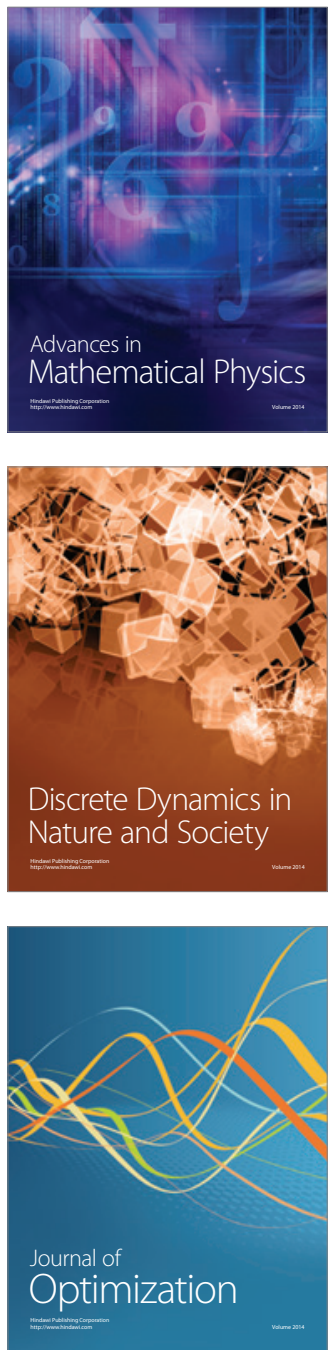